Cochrane Database of Systematic Reviews

\title{
Ab interno trabecular bypass surgery with Trabectome for open- angle glaucoma (Review)
}

Hu K, Shah A, Virgili G, Bunce C, Gazzard G

Hu K, Shah A, Virgili G, Bunce C, Gazzard G.

Ab interno trabecular bypass surgery with Trabectome for open-angle glaucoma.

Cochrane Database of Systematic Reviews 2021, Issue 2. Art. No.: CD011693.

DOI: 10.1002/14651858.CD011693.pub3.

www.cochranelibrary.com 
TABLE OF CONTENTS

HEADER 1

ABSTRACT

PLAIN LANGUAGE SUMMARY

SUMMARY OF FINDINGS

BACKGROUND

OBJECTIVES

METHODS

RESULTS

Figure 1.

Figure 2.

Figure 3.

DISCUSSION

AUTHORS' CONCLUSIONS

ACKNOWLEDGEMENTS

REFERENCES

CHARACTERISTICS OF STUDIES

DATA AND ANALYSES

Analysis 1.1. Comparison 1: phaco-AIT versus phaco-Trab, Outcome 1: Proportion of participants who were medication-free at 12 months

Analysis 1.2. Comparison 1: phaco-AIT versus phaco-Trab, Outcome 2: Mean change in IOP at 12 months

Analysis 1.3. Comparison 1: phaco-AIT versus phaco-Trab, Outcome 3: Mean change in number of IOP-lowering medications per day

Analysis 1.4. Comparison 1: phaco-AIT versus phaco-Trab, Outcome 4: Proportion of participants who achieved an IOP of 14 $\mathrm{mmHg}$ or less at 12 months

Analysis 1.5. Comparison 1: phaco-AIT versus phaco-Trab, Outcome 5: Proportion of participants who achieved an IOP of 17 $\mathrm{mmHg}$ or less at 12 months

Analysis 1.6. Comparison 1: phaco-AIT versus phaco-Trab, Outcome 6: Proportion of participants who achieved an IOP of 21 $\mathrm{mmHg}$ or less at 12 months

Analysis 1.7. Comparison 1: phaco-AIT versus phaco-Trab, Outcome 7: Proportion of participants who required further glaucoma surgery, including laser, at 12 months

Analysis 1.8. Comparison 1: phaco-AIT versus phaco-Trab, Outcome 8: Proportion of participants experiencing any intra- and postoperative complications

Analysis 1.9. Comparison 1: phaco-AIT versus phaco-Trab, Outcome 9: Proportion of participants experiencing intra- and postoperative complications within 30 days postoperative

Analysis 1.10. Comparison 1: phaco-AIT versus phaco-Trab, Outcome 10: Proportion of participants experiencing intra- and postoperative complications $>30$ days postoperative

APPENDICES

WHAT'S NEW

HISTORY

CONTRIBUTIONS OF AUTHORS

DECLARATIONS OF INTEREST

SOURCES OF SUPPORT

DIFFERENCES BETWEEN PROTOCOL AND REVIEW

INDEX TERMS 
[Intervention Review]

\section{Ab interno trabecular bypass surgery with Trabectome for open-angle glaucoma}

Kuang Huํㄹ Anupa Shah², Gianni Virgili3,4, Catey Bunce ${ }^{5}$, Gus Gazzard ${ }^{1}$

1Glaucoma Service, Moorfields Eye Hospital NHS Foundation Trust, London, UK. ${ }^{2}$ Cochrane Eyes and Vision, ICEH, London School of Hygiene \& Tropical Medicine, London, UK. ${ }^{3}$ Department of Neurosciences, Psychology, Drug Research and Child Health (NEUROFARBA), University of Florence, Florence, Italy. ${ }^{4}$ Centre for Public Health, Queen's University Belfast, Belfast, UK. ${ }^{5}$ London, UK

Contact address: Kuang Hu, kuang.hu1@nhs.net.

Editorial group: Cochrane Eyes and Vision Group.

Publication status and date: New search for studies and content updated (conclusions changed), published in Issue 2, 2021.

Citation: Hu K, Shah A, Virgili G, Bunce C, Gazzard G. Ab interno trabecular bypass surgery with Trabectome for open-angle glaucoma. Cochrane Database of Systematic Reviews 2021, Issue 2. Art. No.: CD011693. DOI: 10.1002/14651858.CD011693.pub3.

Copyright (c 2021 The Cochrane Collaboration. Published by John Wiley \& Sons, Ltd.

\section{A B S T R A C T}

\section{Background}

Glaucoma is the leading cause of irreversible blindness. Minimally invasive surgical techniques, such as ab interno trabecular bypass surgery, have been introduced to prevent glaucoma from progressing.

\section{Objectives}

In light of the potential benefits for people with open-angle glaucoma and the widespread uptake of the technique, it is important to critically evaluate the evidence for whether treatment with ab interno trabecular bypass surgery with Trabectome is both efficacious and safe.

\section{Search methods}

We searched the Cochrane Central Register of Controlled Trials (CENTRAL; which contains the Cochrane Eyes and Vision Trials Register; 2020, Issue 7); Ovid MEDLINE; Ovid Embase; the ISRCTN registry; ClinicalTrials.gov and the WHO ICTRP. The date of the search was 17 July 2020.

\section{Selection criteria}

We searched for randomised controlled trials (RCTs) of ab interno trabecular bypass surgery with Trabectome compared to other surgical treatments (other minimally invasive glaucoma device techniques, trabeculectomy), laser treatment, or medical treatment. We also included trials in which these devices were combined with phacoemulsification compared to phacoemulsification in combination with other glaucoma surgery or alone.

\section{Data collection and analysis}

We used the standard methodological procedures expected by Cochrane. Our primary outcome was proportion of participants who were medication-free (not using eye drops). Secondary outcomes included mean change in intraocular pressure (IOP), proportion of participants who required further glaucoma surgery, mean change in quality of life, proportion of participants who achieved an IOP of $21 \mathrm{mmHg}$ or less, $17 \mathrm{mmHg}$ or less, or $14 \mathrm{mmHg}$ or less and rate of visual field progression. Adverse effects were the proportion of participants experiencing intra- and postoperative complications. All outcomes were measured in the short term (6 to 18 months), medium term (18 to 36 months), and long term (36 months or longer). 


\section{Main results}

In this update, we included one RCT which had previously been identified as an ongoing study in our 2016 publication. This trial was a single-centre, single-surgeon RCT set in Canada with 19 participants. Participants were adults who had open-angle glaucoma, open angles, and had inadequately controlled IOP that required surgical intervention. The study was terminated before the intended sample size was reached 'due to slow recruitment and increasing lack of clinical equipoise over time'. This reduced the power of the study to detect clinically important effects. We assessed the trial as being at high risk of attrition, reporting, and other potential sources of biases. The risks of performance and detection bias are unclear.

The intervention group of 10 people had Trabectome ab interno trabeculotomy combined with cataract extraction (phaco-AIT) and the comparator group of 9 people had trabeculectomy with mitomycin C combined with cataract extraction (phaco-Trab), one of whom was lost to follow-up. Seven of 10 participants in the phaco-AIT group and 4 of 8 in the phaco-Trab group were medication-free (not using drops) at 12 months (odds ratio (OR) 2.33, 95\% confidence interval ( $\mathrm{Cl}) 0.34$ to 16.2 ; very low-certainty evidence). At 12 months, the mean change in IOP was worse for phaco-AIT than for phaco-Trab, but this evidence was very uncertain (mean difference (MD) $3.70 \mathrm{mmHg}, 95 \%$ $\mathrm{Cl}-1.44$ to 8.84; very low-certainty evidence) in the phaco-AIT group, as was the difference in the mean number of IOP-lowering drops taken per day (MD - $0.41,95 \% \mathrm{Cl}-1.22$ to 0.40 ; very low-certainty evidence).

Only one participant in the phaco-AIT group required further glaucoma surgery. The study protocol declared that quality of life and visual field progression were measured, but they were not reported

All 8 participants with complete data in the phaco-Trab group and 8 of 10 in the phaco-AIT had at least one early or late postoperative complication (e.g. day 1 IOP spike, hypotony, choroidal effusion, bleb leak or encapsulation, uveitis, or peripheral anterior synechiae).

The evidence was very low-certainty due to high risk of bias for several domains for this study and for large imprecision of all estimates.

We also identified one ongoing study, identified from the International Clinical Trials Registry Platform (ICTRP): a multicentre, open, RCT comparing Trabectome to ab interno trabeculectomy using microhook. The study investigators plan to recruit 120 adults between 20 and 90 years of age. The primary outcome is duration of treatment success. Secondary outcomes include postoperative IOP, number of antiglaucoma medications, and adverse events.

\section{Authors' conclusions}

There is currently no high-quality evidence for the outcomes of ab interno trabecular bypass surgery with Trabectome for open-angle glaucoma. Properly designed RCTs are needed to assess the long-term efficacy and safety of this technique.

\section{PLAIN LANGUAGE SUMMARY}

\section{What are the benefits and risks of minimally invasive (Trabectome) surgery for treating glaucoma (a common eye condition)?}

\section{Why is this question important?}

Glaucoma is a common eye condition that affects the optic nerve. It can occur in people of all ages but is most common in adults over 70 years of age.

There are several different types of glaucoma. The most common is 'primary open-angle glaucoma'. This tends to develop slowly over many years. It is caused by abnormally high pressure in the eye that develops when the eye's drainage system does not work properly and fluid builds up in the eye. This increased pressure damages the optic nerve and can cause partial - or even total - blindness.

Several different treatments are available for glaucoma. These include eye drops, laser treatment, and surgery. Surgery is usually used only if treatment with eye drops or laser has not worked.

Standard surgery usually involves removing part of the drainage tubes in the eye to allow fluid to drain more easily. It may be performed under local anaesthetic (while the patient is awake) or under general anaesthetic (while the patient is asleep). Standard surgery often leads to complications (such as bleeding in the eye during and after surgery), and people may need the operation to be repeated.

One possible alternative to standard surgical procedures is Trabectome surgery. Trabectome surgery is performed under local anaesthetic and involves using a specially designed instrument (a Trabectome) to remove a portion of tissue to improve the eye's drainage pathway. Surgeons make a smaller incision in the eye than in standard surgery, and this could lead to fewer risks for the patient.

To find out about the benefits and risks of Trabectome surgery, we reviewed the evidence from research studies.

\section{How did we identify and evaluate the evidence?}

First, we searched for randomised controlled studies (clinical studies in which people are randomly put into one of two or more treatment groups), because these studies provide the most robust evidence about the effects of a treatment. We then compared the results and summarised the evidence from all the studies. Finally, we rated our confidence in the evidence-based on factors such as study methods and sizes and the consistency of findings across studies. 


\section{What did we find?}

We found one study that involved a total of 19 people with glaucoma. The study was set in Canada and funded by the University of Alberta. People were followed for up to one year after surgery. All participants were operated on by the same surgeon. Trabectome surgery was compared against a standard surgical procedure (trabeculectomy).

Based on the single study we found, we cannot determine whether Trabectome surgery is better or worse than conventional surgery. The study was very small, and there were problems with the way it was conducted. Therefore, we have too little confidence in its results about the following:

- the need to use any, or fewer, eye drops one year after surgery;

- improvement in eye pressure one year after surgery;

- the need for more surgery; and

- complications.

Other aspects, such as changes in vision or quality of life, were not studied.

We identified one ongoing study that is being conducted in Japan. Once results are available for this study, it will be possible to add them to future versions of this review.

\section{What does this mean?}

We do not know whether Trabectome surgery is better or worse than other treatments for glaucoma. This is because there is too little evidence at present. It would be helpful if more studies were conducted in this area in future. In particular, there is a need for robust studies that assess the long-term benefits and risks of Trabectome surgery.

\section{How-up-to date is this review?}

The evidence in this Cochrane Review is current to July 2020. 


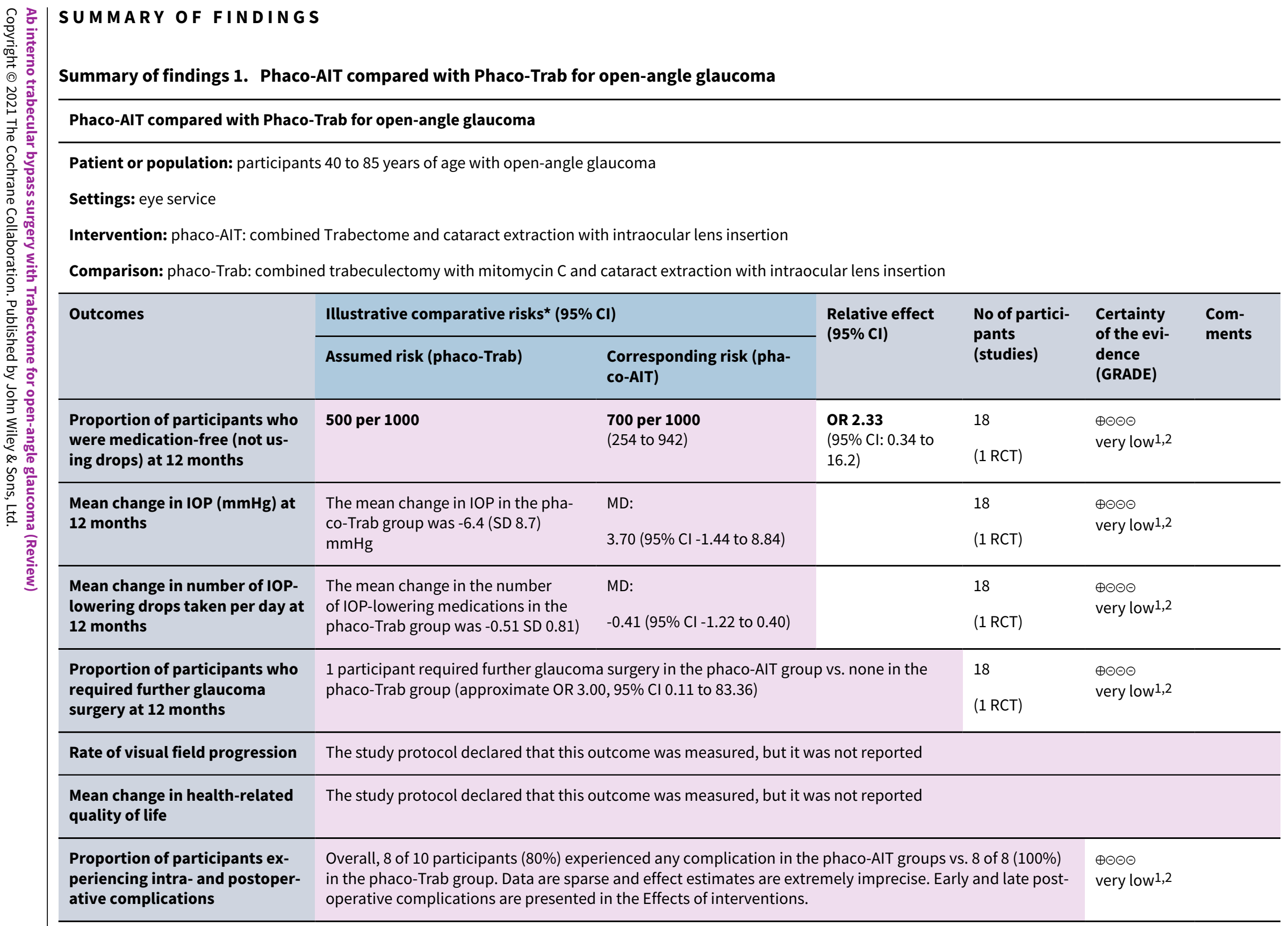


*The assumed risk is the median risk in control groups in the included studies (rounded to nearest 10 per 1000). The corresponding risk (and its $95 \% \mathrm{Cl}$ ) is based on the assumed risk in the comparison group and the relative effect of the intervention (and its $95 \% \mathrm{Cl}$ ).

CI: confidence interval; IOP: intraocular pressure; MD: mean difference; OR: odds ratio; RCT: randomised controlled trial

\section{GRADE Working Group grades of evidence}

High-certainty: We are very confident that the true effect lies close to that of the estimate of the effect.

Moderate-certainty: We are moderately confident in the effect estimate: the true effect is likely to be close to the estimate of the effect, but there is a possibility that it is substantially different.

Low-certainty: Our confidence in the effect estimate is limited: the true effect may be substantially different from the estimate of the effect.

Very low-certainty: We have very little confidence in the effect estimate: the true effect is likely to be substantially different from the estimate of effect.

1) downgraded -1 level for serious limitations in study design

2) downgraded -2 level for imprecision 


\section{B A C K G R O U N D}

\section{Description of the condition}

Glaucoma is a chronic progressive optic neuropathy affecting up to $4 \%$ of people by 80 years of age (Burr 2007). It is the leading cause of irreversible blindness, affecting 60 million people globally (Quigley 2006). This figure is expected to increase to 80 million people by 2020. Open-angle glaucoma (OAG) is the most common type, accounting for three-quarters of cases (Quigley 2006). In one large population cohort, one in six people with OAG became bilaterally blind (Peters 2013). The only proven way to prevent vision loss is to reduce the pressure inside the eye (intraocular pressure (IOP)) over the long term (AGIS 2000; CNTG Study Group 1998; Heijl 2002; Kass 2002). Approaches to reducing IOP include medical therapy, laser treatments, and surgery. As commercially available eye drop preparations have a short-lasting effect, medical therapy requires eye drops to be instilled one or more times daily for life. Adherence is very poor, even if use is monitored (Friedman 2009; Okeke 2009). Conventional surgical techniques such as trabeculectomy are associated with significant risks, with more than $40 \%$ of patients developing perioperative complications (Kirwan 2013; Lichter 2001), and $7 \%$ to $18 \%$ of cases requiring re-operation (Gedde 2012; Kirwan 2013). These techniques are therefore often reserved for disease that is progressing despite other treatments (King 2013).

\section{Description of the intervention}

A number of minimally invasive surgical techniques have recently been developed with the aim of achieving long-term reduction of IOP with a better safety profile than conventional surgery (Francis 2011). Among them, ab interno trabecular bypass surgery (also known as trabeculotomy ab interno and trabeculectomy ab interno) with the Trabectome (NeoMedix, Tustin, CA, USA) is a Food and Drug Administration (FDA)-approved and CE marked treatment.

\section{How the intervention might work}

The trabecular meshwork is the eye's main site of resistance to outflow of aqueous humour (Overby 2009). The Trabectome is designed to selectively ablate a portion of the trabecular meshwork, enabling aqueous humour to have direct access to the canal of Schlemm and thence the collector channels (Francis 2006). This is intended to promote aqueous outflow, thereby reducing IOP. Tissue ablation is performed electrosurgically using a 19.5-gauge instrument, which is introduced into the eye via a $1.6-\mathrm{mm}$ incision in the cornea.

\section{Why it is important to do this review}

Consultation with patients and healthcare professionals has identified a need for better treatments for glaucoma (James Lind Alliance 2013). Minimally invasive glaucoma procedures may safely and effectively reduce IOP in the long term, removing concerns about permanent vision loss due to nonadherence to eye drops. A single treatment may also be more acceptable to patients than daily and indefinite self-administration of eye drops. Initial results of ab interno trabecular bypass surgery with the Trabectome were reported in 2005 (Minckler 2005). Post-market surveillance data show that more than 4600 treatments were performed between 2004 and 2013 at over 200 centres worldwide (Mosaed 2014; NeoMedix, on file). In light of the potential benefits for patients and the widespread uptake of the technique, it is important to critically evaluate the evidence for whether treatment with the Trabectome is both efficacious and safe. Importantly, Trabectome surgery may be combined with phacoemulsification (cataract surgery), a sight-restoring operation to remove the natural lens of the eye when it has lost clarity. Since phacoemulsification itself reduces IOP (Mansberger 2012), we planned specifically to examine the evidence for efficacy of Trabectome treatment in people who have concomitant phacoemulsification compared to those who do not.

This Cochrane Review is part of a suite of reviews on minimally invasive glaucoma surgery (MIGS) techniques and devices currently being undertaken by the Cochrane Eyes and Vision MIGS Consortium, which includes Hydrus Schlemm's canal Microstent (Ivantis Inc., Irvine, CA, USA) (Otarola 2017), endoscopic cytophotocoagulation (Endo Optiks, Waltham, MA, USA) (Tóth 2017), XEN Glaucoma Implant (AqueSys Implant, Aliso Viejo, CA, USA) (King 2018), iStent and iStent inject (Glaukos Corporation, Laguna Hills, CA, USA) (Le 2019), and supraciliary microstent surgery (Sandhu 2017).

\section{OBJECTIVES}

In light of the potential benefits for people with open-angle glaucoma and the widespread uptake of the technique, it is important to critically evaluate the evidence for whether treatment with ab interno trabecular bypass surgery with Trabectome is both efficacious and safe.

\section{METHODS}

\section{Criteria for considering studies for this review}

\section{Types of studies}

We included randomised controlled trials (RCTs) only. We included all study reports published in English (or which have been translated into English), irrespective of their publication status. We planned to exclude within-person studies because of the potential for medical treatments, such as topical beta blockers, used for one eye to influence the outcome in the other eye (Piltz 2000).

\section{Types of participants}

Participants could have OAG of any type, including primary and secondary OAG. We excluded closed-angle glaucoma. As there are no universally accepted criteria by which glaucoma may be defined, we permitted studies to use their own definitions of glaucoma (provided these were clearly stated). We also included participants with ocular hypertension, normal-tension glaucoma, or possible glaucoma (suspects for glaucoma). We applied no restrictions regarding location, setting, or demographic factors.

\section{Types of interventions}

The intervention was ab interno trabeculotomy performed with the Trabectome (NeoMedix, Tustin, CA, USA). Although it is possible to ablate a variable amount of the trabecular meshwork (typically an arc of $40^{\circ}$ ) and to vary the electrosurgical power employed (Francis 2006), we did not apply any particular inclusion or exclusion criteria to these or other treatment delivery parameters.

We compared ab interno trabeculotomy performed with the Trabectome to the following: 
- laser treatment (selective laser trabeculoplasty or argon laser trabeculoplasty);

- other MIGS techniques;

- conventional glaucoma surgery (trabeculectomy);

- medical therapy.

We also included trials in which these devices were combined with phacoemulsification compared to phacoemulsification in combination with other glaucoma surgery or alone.

\section{Types of outcome measures}

We did not use the reporting of particular outcomes as a criterion for eligibility for this review. We did not exclude studies from this review solely on the grounds of an outcome of interest not being reported.

We reported outcomes in the short term (6 to 18 months), medium term (18 to 36 months), and long term (36 months or longer).

\section{Primary outcomes}

- Proportion of participants who were medication-free (not using eye drops).

Several different glaucoma outcome measures have been specified as primary outcomes in other Cochrane Reviews and Protocols (Ismail 2015). A recent study classified IOP, visual field, safety, and anatomic outcomes as being highly important to glaucoma experts (Ismail 2016). A panel of patients from the Patient and Public Involvement Group of the National Institute for Health Research (NIHR) Biomedical Research Centre for Ophthalmology identified drop-free disease control as a highly valued outcome (unpublished). We chose a patient-centred primary outcome. In assessing this effect measure, we planned to report how prescribing IOP-lowering eye drops was determined during followup. We planned to examine whether the people measuring IOP and those deciding on prescribing IOP-lowering eye drops were masked to the treatment group.

\section{Secondary outcomes}

- Mean change in IOP measured using Goldmann applanation tonometry.

- Mean change in number of IOP-lowering drops taken per day.

- Proportion of participants who achieve an IOP of $21 \mathrm{mmHg}$ or less.

- Proportion of participants who achieve an IOP of $17 \mathrm{mmHg}$ or less.

- Proportion of participants who achieve an IOP of $14 \mathrm{mmHg}$ or less.

- Proportion of participants who required further glaucoma surgery, including laser, as recorded by the investigators of the included trial.

- Rate of visual field progression (decibels (dB)/time) or proportion of participants whose field loss progressed in the follow-up period.

- Mean change in HRQoL.

\section{Adverse effects}

- Proportion of participants experiencing intra- and postoperative complications, including, but not restricted to, the following:
- loss of visual acuity (more than 2 Snellen lines or more than $0.3 \log$ MAR, according to the method of recording visual acuity; or loss of light perception);

- bleeding, as recorded by the investigators;

- endophthalmitis, as recorded by the investigators;

- IOP spikes (postoperative rise in IOP, measured using Goldmann applanation tonometry, of more than 10 $\mathrm{mmHg}$ compared to the previous assessment, including measurements taken during the first postoperative month).

\section{Search methods for identification of studies}

\section{Electronic searches}

The Cochrane Eyes and Vision Information Specialist conducted systematic searches in the following databases for randomised controlled trials and controlled clinical trials. There were no restrictions to language or year of publication. The date of the search was 17 July 2020.

- Cochrane Central Register of Controlled Trials (CENTRAL; 2020, Issue 7) (which contains the Cochrane Eyes and Vision Trials Register) in the Cochrane Library (searched 17 July 2020) (Appendix 1).

- MEDLINE Ovid (1946 to 17 July 2020) (Appendix 2).

- Embase Ovid (1980 to 17 July 2020) (Appendix 3).

- ISRCTN registry (www.isrctn.com/editAdvancedSearch; searched 17 July 2020) (Appendix 4).

- US National Institutes of Health Ongoing Trials Register ClinicalTrials.gov (www.clinicaltrials.gov; searched 17 July 2020) (Appendix 5).

- World Health Organization (WHO) International Clinical Trials Registry Platform (ICTRP) (www.who.int/ictrp; searched 17 July 2020) (Appendix 6).

\section{Searching other resources}

We searched the reference lists of the included study for other possible studies, and we planned to contact any individuals or organisations we believed may have conducted or be conducting relevant RCTs. We also searched the website of the manufacturer (NeoMedix Inc., Tustin, CA, USA; www.trabectome.com) for any information on forthcoming trials.

\section{Data collection and analysis}

\section{Selection of studies}

For the 2021 update, two review authors (AS, KH) independently screened titles and abstracts of all articles identified by the search. If abstracts were not available, we planned to screen full-text articles. Two review authors (AS, KH) independently assessed full-text reports of all potentially eligible studies. In case of disagreement regarding eligibility, a third review author would arbitrate. If we rejected any full-text reports, we planned to record the reasons for this.

\section{Data extraction and management}

Two review authors (AS, KH) independently extracted study characteristics from reports of each study and entered the data into Review Manager 5 (RevMan 5) (Review Manager 2014). Two authors $(\mathrm{KH}, \mathrm{GV})$ independently extracted the data for the analyses. 
Data collected in Appendix 7 was presented in the 'Characteristics of included studies' table.

If data on included studies were missing or unclear, we planned to contact the individuals or organisations involved to obtain clarification. We intended to collect and use the most detailed numerical data available to facilitate analyses of included studies. We planned to obtain these data from individuals or organisations instead of using less precise methods, such as extracting numeric data from graphs. If this was necessary, two review authors would independently extract the data, and a third review author would arbitrate in case of disagreement.

\section{Assessment of risk of bias in included studies}

We used the original Cochrane 'Risk of bias' tool (RoB 1) as described in Chapter 8 of the Cochrane Handbook for Systematic Reviews of Interventions to assess the risk of bias and assign judgements of this for included studies (Higgins 2017).

\section{Measures of treatment effect}

We reported outcomes in the short term ( 6 to 18 months), medium term (18 to 36 months), and long term (36 months or longer) after randomisation.

The primary outcome was the proportion of participants who were medication-free. We used the odds ratio (OR) as the treatment effect measure.

We reported mean change in IOP and mean change in number of IOP-lowering drops taken per day. We reported the proportion of participants achieving various target IOPs or requiring further glaucoma surgery using ORs as the treatment effect measure. We planned to report HRQoL outcomes as differences in means or ORs for continuous and binary data, respectively. We reported secondary safety outcomes as ORs.

\section{Unit of analysis issues}

We noted whether the studies included one or two eyes from each participant and whether randomisation had been conducted at the level of the participant or the eye. There is a potential for medical treatments, such as topical beta blockers, used for one eye to influence the outcome in the other eye (Piltz 2000). We therefore planned to exclude studies that adopted a paired design. Surgery to lower IOP in one eye may also affect the IOP of the other eye (Radcliffe 2010).

\section{Dealing with missing data}

We planned to minimise missing outcome data by contacting individuals and organisations to try to obtain them. If the data were unavailable, but the level of missing data in each group and reasons for missing data in each group were similar, we may simply analyse available case data if an intention-to-treat (ITT) analysis had not been performed. If the authors had conducted their own ITT analysis despite missing data, we planned to document whether they provided any justification for the method they had used to deal with missing data and whether they had compared their ITT result with an available case result.

\section{Assessment of heterogeneity}

We planned to assess heterogeneity between trials by careful examination of the study reports, assessing forest plots, and an examination of the $1^{2}$ value with its $95 \%$ confidence interval. We would consider $\mathrm{I}^{2}$ values greater than $50 \%$ as indicating substantial heterogeneity and, therefore, suggesting that metaanalysis might not be wise, however, we would give consideration to the consistency of the effect estimates. If all estimates were in the same direction, we might meta-analyse, even where heterogeneity was evident, and comment on the heterogeneity.

\section{Assessment of reporting biases}

We planned to use a funnel plot to assess the risk of publication bias if we included more than 10 trials in the review.

\section{Data synthesis}

We planned to undertake a meta-analysis where data appeared clinically, methodologically, and statistically homogeneous. We would check that participants, interventions, comparators, and outcomes were sufficiently similar to give a clinically meaningful result and that our $1^{2}$ value result indicated little inconsistency (that is $1^{2}<50 \%$ ). If all estimates were in the same direction, we might meta-analyse even where heterogeneity was evident but would comment on this. We planned to use a random-effects model unless there were fewer than three eligible studies, in which case we would use a fixed-effect model.

\section{Subgroup analysis and investigation of heterogeneity}

In future updates of this review, we do not plan to conduct subgroup analysis.

\section{Sensitivity analysis}

We planned to assess the impact of including studies at high risk of bias.

\section{Summary of findings and assessment of the certainty of the evidence}

We prepared a table to summarise the findings of the review, including the assessment of the quality of evidence for all outcomes using the GRADE approach (Langendam 2013).

We reported on the following outcomes at short-term follow-up (6 to 18 months) in 'Summary of findings 1 ' for each comparison listed in the Types of interventions:

- proportion of participants who were medication-free (not using eye drops);

- mean change in IOP, measured using Goldmann applanation tonometry;

- mean change in number of IOP-lowering drops taken per day;

- proportion of participants who required further glaucoma surgery, including laser;

- rate of visual field progression ( $\mathrm{dB} /$ time) or proportion of participants whose field loss progressed in the follow-up period;

- mean change in HRQoL;

- proportion of participants experiencing intra- and postoperative complications (any time point). 


\section{RESULTS}

\section{Description of studies}

\section{Results of the search}

The electronic searches run in 2016 yielded a total of 155 references. The Cochrane Information Specialist removed 41 duplicate records, and two review authors (GG and $\mathrm{KH}$ ) independently screened the remaining 114 reports for potentially eligible studies, namely possible RCTs.
Update searches run in July 2020 yielded a further 268 records (Figure 1). The Cochrane Information Specialist removed 107 duplicates, and we screened the remaining 161 references. We obtained three full-text reports for further assessment. We included one new study (Ting 2018) and excluded one ongoing study (NCT03894631). We have identified a new ongoing study (JPRNUMIN000030167) and will assess this for inclusion when data become available.

Figure 1. Study flow diagram.
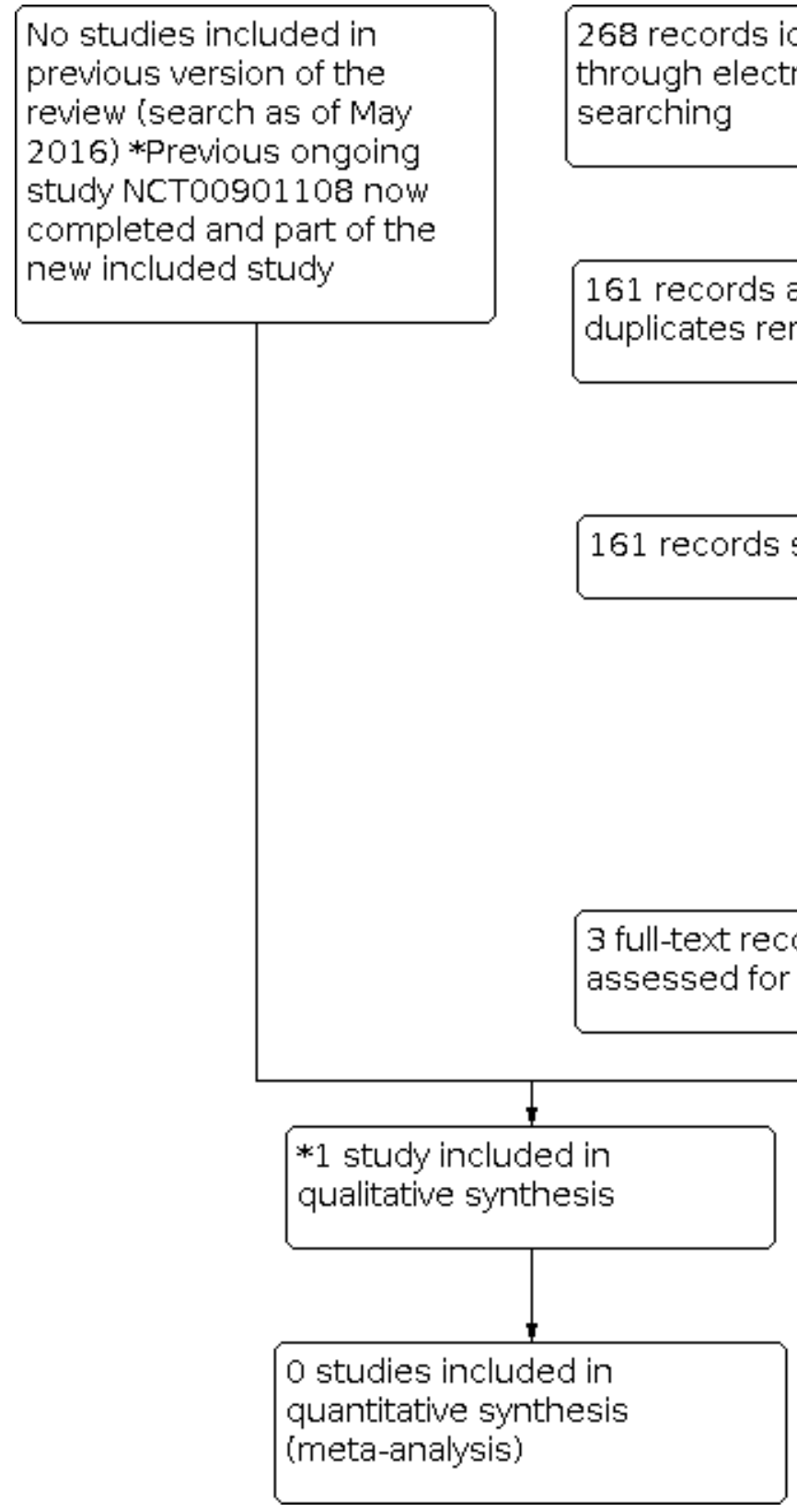

We are not aware of any other individuals or organisations who have conducted or may be conducting relevant RCTs. A search of the website of the manufacturer (NeoMedix Inc., Tustin, CA,

\section{8 records identified} through electronic database searching

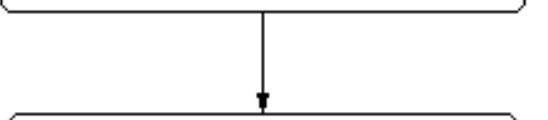

161 records after

duplicates removed

158 records excluded

\section{1 ongoing trial} excluded with reasons

1 ongoing study to be assessed when data become available
USA; www.trabectome.com) did not yield any information on forthcoming trials. 


\section{Included studies}

The 2021 update included one published trial that met our inclusion criteria (Ting 2018), which was an ongoing study in the previous published version of this review (Hu 2016).

\section{Design: prospective RCT}

Sample sizes: 19 (10 phaco-Trabectome, 9 phaco-trabeculectomy); the original protocol described a planned sample size of 52 (26 per study arm), however, the trial report describes a planned sample size of 26 (13 per group)

\section{Setting: eye service}

Participants: people with open-angle glaucoma

Interventions: phaco-Trabectome (phaco-ab interno trabeculectomy, phaco-AIT) versus phaco-trabeculectomy (phacoTrab) with mitomycin C

Outcomes: primary outcomes were mean IOP at 6 months and surgical complications; secondary outcomes were mean IOP at 12 months, achievement of target IOP, number of glaucoma medications, and rate of secondary surgery

\section{Excluded studies}

We excluded one ongoing study (NCT03894631), as both the intervention and comparator were in the same participant, and per our protocol (Hu 2015), we excluded these studies from the outset.

\section{Ongoing studies}

In the 2021 update, we identified one ongoing study that met our inclusion criteria (JPRN-UMIN000030167). The study started in April 2018 and is being conducted in Japan. It is a multicentre RCT with no masking of participants, personnel, or outcome assessors. It compares Trabectome to ab interno trabeculectomy using microhook. Participants will be between 20 and 90 years of age, male and female, and sample size is set at 120 people. The study includes participants with ocular hypertension and openangle glaucoma with IOPs of 20 to $39 \mathrm{mmHg}$ while on treatment with at least one antiglaucoma medication. The primary outcome is duration of treatment success (defined as IOP 5 to $21 \mathrm{mmHg}$, a $20 \%$ reduction from baseline, and no additional glaucoma medical therapy or additional glaucoma surgery). Secondary outcomes include postoperative IOP, number of antiglaucoma medications, and adverse events. See Characteristics of ongoing studies for further information.

\section{Risk of bias in included studies}

See Figure 2; Figure 3

Figure 2. Risk of bias graph: review authors' judgements about each risk of bias item presented as percentages across all included studies.

Random sequence generation (selection bias)

Allocation concealment (selection bias)

Blinding of participants and personnel (performance bias): All outcomes Blinding of outcome assessment (detection bias): All outcomes Incomplete outcome data (attrition bias): All outcomes Selective reporting (reporting bias)

Other bias

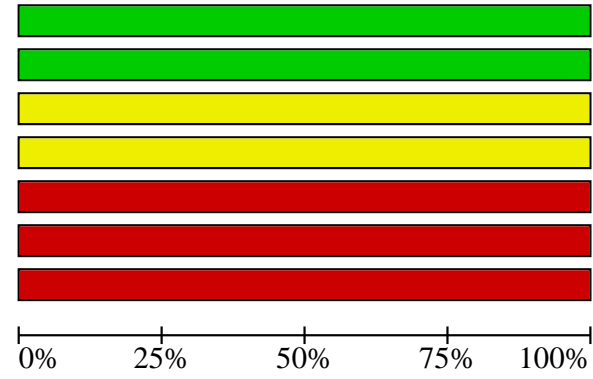


Figure 3. Risk of bias summary: review authors' judgements about each risk of bias item for each included study.

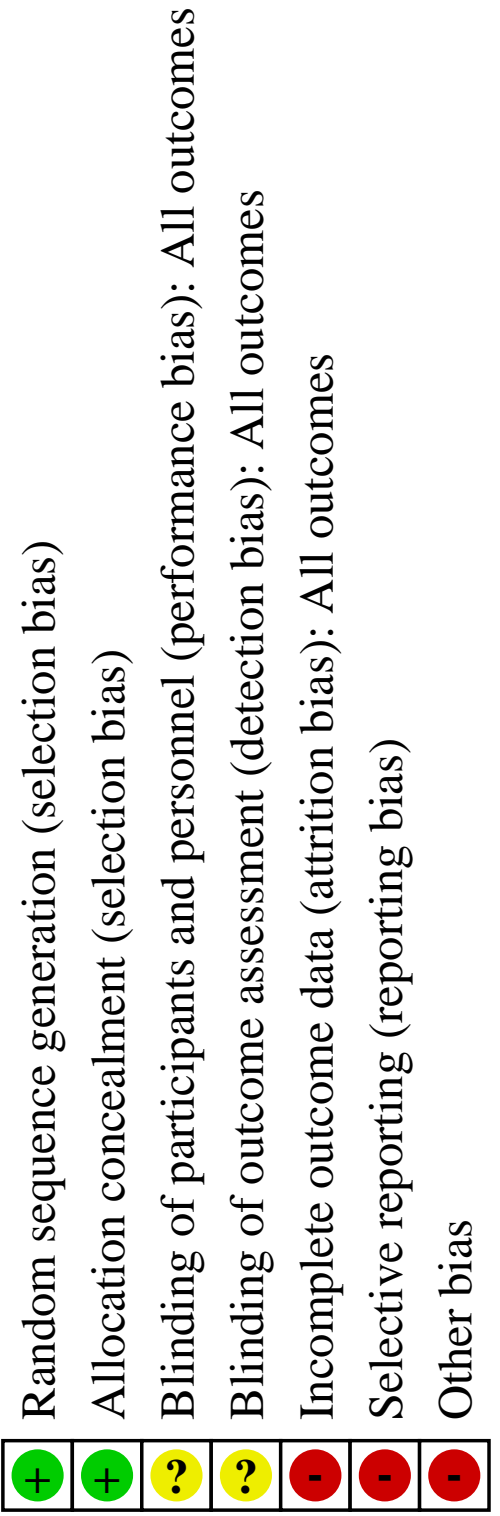

\section{Allocation}

\section{Random sequence generation}

Participants were block randomised to either phaco-AIT or phacoTrab using a randomly permutated block scheme. We assessed the trial to have low risk of selection bias from this source.

\section{Allocation concealment}

Study enrolment and randomisation were performed by a study coordinator to ensure allocation concealment. We assessed the trial to have low risk of selection bias from this source. 


\section{Blinding}

\section{Performance bias}

As the trial report did not mention masking of participants and personnel, we assessed the trial to have unclear risk of performance bias.

\section{Detection bias}

As the trial report did not mention masking of outcome assessors, we assessed the trial to have unclear risk of detection bias.

\section{Incomplete outcome data}

One of nine participants in the phaco-Trab group was lost to followup by 6 months and did not return at 12 months. One of the 10 participants in the phaco-AIT group missed follow-up at 6 months but returned to follow-up at 12 months. We assessed the trial to have high risk of attrition bias.

\section{Selective reporting}

We planned to report quality of life measures and visual acuity according to the original protocol, but we did not include these in the trial report. We reported achievement of target, but we did not include this outcome measure in the protocol. We assessed the trial to be at high risk of reporting bias.

\section{Other potential sources of bias}

The original protocol described a planned sample size of 52 (26 per study arm). However, the trial report describes a planned sample size of 26 (13 per group). It is unclear why the planned sample size changed.

The study was terminated before the intended sample size was reached 'due to slow recruitment and increasing lack of clinical equipoise over time'. The authors state, 'it became more apparent during the study that there was limited overlap between patients suitable for AIT and conventional trabeculectomy'. This reduces the power of the study to detect statistically significant differences.

We note that modifications to ocular hypotensive medications were made at the discretion of the surgeon, not according to a prespecified protocol. This could introduce bias if the surgeon was not masked to the treatment group.

Overall, we assessed the trial to be at high risk of other potential sources of bias.

\section{Effects of interventions}

See: Summary of findings 1 Phaco-AIT compared with Phaco-Trab for open-angle glaucoma

\section{Phaco-AIT versus phaco-Trab}

In Ting 2018, several outcomes of our review were not analysed, but we have been able to calculate the outcomes from the data presented in the article. See Summary of findings 1 . The study included 19 participants (10 phaco-AIT and 9 phaco-Trab). One participant was lost to follow-up after three months.

\section{Proportion of participants who were medication-free (not using eye drops)}

The following table presents the proportion of participants who were medication-free at baseline and during follow-up. At 12 months, we found no evidence of a difference between phaco-AIT and phaco-Trab at 12 months (Analysis 1.1), but estimates were very imprecise (OR: 2.33 , 95\% confidence interval $(\mathrm{Cl}) 0.34$ to 16.2 ; very low-certainty of evidence, downgraded $(-1)$ for risk of bias and (-2) for imprecision).

\begin{tabular}{lllll}
\hline & phaco-AIT & & phaco-Trab \\
\hline Time point & $\mathbf{n}(\%)$ & Total & $\mathbf{n}(\%)$ & Total \\
\hline baseline & $2(20 \%)$ & 10 & $2(22 \%)$ & 9 \\
\hline $\mathbf{6}$ months & $6(67 \%)$ & 9 & $6(75 \%)$ & 8 \\
\hline $\mathbf{1 2}$ months & $7(70 \%)$ & 10 & $4(50 \%)$ & 8 \\
\hline
\end{tabular}

\section{Mean change in IOP measured using Goldmann applanation tonometry}

Both techniques reduced IOP compared to baseline (Analysis 1.2). At 12 months, the MD in the mean IOP change was $3.70 \mathrm{mmHg}$ ( $95 \% \mathrm{Cl}-1.44$ to 8.84$)$, favouring phaco-Trab, but this evidence was very uncertain (very low-certainty of evidence, downgraded (-1) for risk of bias and (-2) for imprecision). In this analysis, the SD of the difference in IOP change was not available, and we computed it using SDs of baseline and final values, assuming a correlation of 0.7 between these measures.

\section{Mean change in number of IOP-lowering drops taken per day}

At 12 months, there was no evidence of a difference in the mean change in IOP-lowering drops at 12 months for phaco-AIT versus phaco-Trab (MD - $0.41,95 \% \mathrm{Cl}-1.22$ to 0.40 ; Analysis 1.3; very lowcertainty of evidence, downgraded (-1) for risk of bias and $(-2)$ for imprecision).

\section{Proportion of participants who achieved an IOP of $21 \mathrm{mmHg}, 17$ $\mathrm{mmHg}, 14 \mathrm{mmHg}$, or less}

The following table presents the proportion of participants achieving different targets of IOP at baseline and during follow-up. At 12 months, the OR $(95 \% \mathrm{Cl})$ of the proportion of participants with IOP of $21,17,14 \mathrm{mmHg}$ or less were 1.29 (0.07 to 24.38; Analysis 
1.4), 0.90 ( 0.13 to 6.08 ; Analysis 1.5 ), and 0.19 (0.01 to 2.29; Analysis 1.6), meaning that no evidence of a difference was found, but estimates were very imprecise due to the limited sample size (very low-certainty of evidence, downgraded (-1) for risk of bias and (-2) for imprecision). Moreover, one participant in the phaco-AIT group received glaucoma surgery at 10 months.

\begin{tabular}{|c|c|c|c|c|c|}
\hline & Intervention & $\leq 21 \mathrm{mmHg}$ & $\leq 17 \mathrm{mmHg}$ & $\leq 14 \mathrm{mmHg}$ & Total \\
\hline \multirow[t]{2}{*}{ baseline } & phaco-AIT & $6(60 \%)$ & $5(50 \%)$ & 0 & 10 \\
\hline & phaco-Trab & $5(56 \%)$ & $1(11 \%)$ & 0 & 9 \\
\hline \multirow[t]{2}{*}{6 months } & phaco-AlT & $7(78 \%)$ & $5(56 \%)$ & $2(22 \%)$ & 9 \\
\hline & phaco-Trab & $7(88 \%)$ & $6(75 \%)$ & $1(13 \%)$ & 8 \\
\hline \multirow{2}{*}{$\begin{array}{l}12 \\
\text { months }\end{array}$} & phaco-AlT & $9(90 \%)$ & $6(60 \%)$ & $1(10 \%)$ & 10 \\
\hline & phaco-Trab & $7(88 \%)$ & $5(63 \%)$ & $3(38 \%)$ & 8 \\
\hline
\end{tabular}

\section{Proportion of participants who required further glaucoma surgery, including laser, as recorded by the investigators of the included trial}

One of 10 participants (10\%) in the Phaco-AlT group required further glaucoma surgery (Ahmed valve at 10 months postoperative). None of the nine participants $(0 \%)$ in the PhacoTrab group remaining under follow-up required further glaucoma surgery. At 12 months, data were sparse and the evidence was very uncertain (OR 3.00, 95\% Cl 0.11 to 83.4 ; Analysis 1.7; very lowcertainty of evidence, downgraded (-1) for risk of bias and $(-2)$ for imprecision).

\section{Mean change in HRQOL}

The study protocol declared that this outcome was measured, but it was not reported.

\section{Rate of visual field progression (dB/time) or proportion of} participants whose field loss progressed in the follow-up period

The study protocol declared that this outcome was measured, but it was not reported.

\section{Proportion of participants experiencing intra- and postoperative complications}

Overall, 8 of 10 participants (80\%) experienced any complication in the phaco-AIT groups versus 8 of $8(100 \%)$ in the phaco-Trab group. Because of sparse data, estimates were very imprecise (OR 0.20 , $95 \% \mathrm{Cl} 0.01$ to 4.82; Analysis 1.8; very low-certainty of evidence, downgraded (-1) for risk of bias and (-2) for imprecision).

We report the following details on early complications (within 30 days postoperative) and late complications ( $>30$ days postoperative), and more than one complication is possible for each participant based on available cases.

\section{Early complications}

Phaco-AIT: 8 of 10 participants (80\%): peripheral anterior synechiae (5), day 1 IOP spike (5), hyphema (4), steroid response (1) as mild or moderate early complications (more than one complication is possible for each participant).
Phaco-Trab: 8 of 8 participants (100\%): peripheral anterior synechiae (1), day 1 IOP spike (3), hypotony (3), bleb leak (2), which were mild or moderate complications; and hypotony maculopathy (2) and choroidal effusion (2) as severe early complications (more than one complication is possible for each participant).

There was no evidence of a difference in the proportion of participants with early complications for phaco-AIT vs phaco-Trab, but estimates were very imprecise $(\mathrm{OR} 0.50,95 \% \mathrm{Cl} 0.04$ to 6.68 ; Analysis 1.9; very low-certainty of evidence, downgraded (-1) for risk of bias and (-2) for imprecision).

\section{Late complications}

Phaco-AIT: 6 of 10 participants (60\%): peripheral anterior synechiae (5), chronic-recurrent uveitis (2) as mild or moderate early complications (more than one complication is possible for each participant).

Phaco-Trab: 3 of 8 participants (38\%): peripheral anterior synechiae (2), chronic-recurrent uveitis (2), encapsulated bleb (1) as mild or moderate complications (more than one complication is possible for each participant).

There was no evidence of a difference in the proportion of participants with late complications for phaco-AIT vs phaco-Trab, but estimates were very imprecise (OR $2.50,95 \% \mathrm{Cl} 0.37$ to 16.9 ; Analysis 1.10; very low-certainty of evidence, downgraded $(-1)$ for risk of bias and (-2) for imprecision).

\section{DISCUSSION}

We found one RCT reporting the outcomes of ab interno trabecular bypass surgery with Trabectome for open-angle glaucoma (Ting 2018) and one RCT that is in progress (JPRN-UMIN000030167).

\section{Summary of main results}

All efficacy and safety estimates obtained in this study were unreliable because of risk of bias and a very small sample size. 


\section{Overall completeness and applicability of evidence}

We believe that our conclusions are supported by a thorough search of available evidence, as outlined in the published protocol (Hu 2015).

\section{Quality of the evidence}

The included study was terminated before the intended sample size was reached 'due to slow recruitment and increasing lack of clinical equipoise over time'. This reduces the power of the study to detect statistically significant differences. We assessed the trial as being at high risk of attrition, reporting, and other biases. The risks of performance and detection bias are unclear. Overall, we judged the evidence to be of very low-certainty.

\section{Potential biases in the review process}

The review authors may not be aware of individuals or organisations that have conducted or may be conducting relevant RCTs, therefore it is possible that relevant RCTs have not been identified.

\section{Agreements and disagreements with other studies or reviews}

A review examining IOP and glaucoma medications following ab interno trabecular bypass surgery with Trabectome was published recently (Kaplowitz 2016). This review included only prospective or retrospective case series or cohorts, so the results are not comparable with our review.

\section{AUTHORS' CONCLUSIONS}

\section{Implications for practice}

There is currently no high-quality evidence for the outcomes of ab interno trabecular bypass surgery with Trabectome for openangle glaucoma. Practitioners need to take this into account when considering treatment options for open-angle glaucoma.

\section{Implications for research}

Ab interno trabecular bypass surgery with Trabectome has been used for over 10 years. Properly designed RCTs are needed to assess the long-term efficacy and safety of this technique compared to conventional glaucoma treatments for people with open-angle glaucoma. These RCTs should assess outcomes that are relevant to patients, such as freedom from using eye drops. If superiority is demonstrated compared to conventional treatments, the next step would be to determine whether Trabectome is superior to other forms of minimally invasive glaucoma surgery.

\section{A CKN OWLEDGEMENTS}

We thank Iris Gordon of Cochrane Eyes and Vision (CEV) for creating and executing the electronic search strategies. We thank Nitin Anand and Jennifer Evans for their comments on the protocol/ review. We thank Richard Wormald for his contributions to the first published version of this review (Hu 2016). We thank Claire Allcock for proofreading the final version of the original published review (Hu 2016).

The 2021 update was signed off for publication by Augusto AzuaraBlanco, Editor for CEV. 


\section{RE F E R E N C E S}

\section{References to studies included in this review}

\section{Ting 2018 \{published data only\}}

Ting JL, Rudnisky CJ, Damji KF. Prospective randomized controlled trial of phaco-trabectome versus phacotrabeculectomy in patients with open angle glaucoma. Canadian Journal of Ophthalmology 2018;53(6):588-94.

\section{References to studies excluded from this review}

NCT03894631 \{published data only\}

NCT03894631. Effect of intra-subject phaco/trabectome vs. phaco/kahook dual blade on intraocular pressure. clinicaltrials.gov/ct2/show/NCT03894631 (first received 28 March 2019).

\section{References to ongoing studies}

JPRN-UMIN000030167 \{published data only\}

JPRN-UMIN000030167. Trabectome versus microhook ab interno trabeculotomy comparative study (TramTrac Study) [A multicenter, randomized, trial to test non-inferiority of microhook ab interno trabeculotomy against Trabectome for an intraocular pressure lowering effect and safety]. upload.umin.ac.jp/cgi-open-bin/ctr_e/ctr_view.cgi? recptno=R000032738 (registered date 27 November 2017).

\section{Additional references}

\section{AGIS 2000}

Anonymous. The Advanced Glaucoma Intervention Study (AGIS): 7. The relationship between control of intraocular pressure and visual field deterioration. The AGIS Investigators. American Journal of Ophthalmology 2000;130(4):429-40.

\section{Burr 2007}

Burr JM, Mowatt G, Hernandez R, Siddiqui MA, Cook J, Lourenco T, et al. The clinical effectiveness and costeffectiveness of screening for open angle glaucoma: a systematic review and economic evaluation. Health Technology Assessment 2007;11(41):iii-iv, ix-x, 1-190.

\section{CNTG Study Group 1998}

Anonymous. Comparison of glaucomatous progression between untreated patients with normal-tension glaucoma and patients with therapeutically reduced intraocular pressures. Collaborative Normal-Tension Glaucoma Study Group. American Journal of Ophthalmology 1998;126(4):487-97.

\section{Francis 2006}

Francis BA, See RF, Rao NA, Minckler DS, Baerveldt G. Ab interno trabeculectomy: development of a novel device (Trabectome) and surgery for open-angle glaucoma. Journal of Glaucoma 2006;15(1):68-73. the American Academy of Ophthalmology. Ophthalmology 2011;118(7):1466-80.

\section{Friedman 2009}

Friedman DS, Okeke CO, Jampel HD, Ying GS, Plyler RJ, Jiang Y, et al. Risk factors for poor adherence to eyedrops in electronically monitored patients with glaucoma. Ophthalmology 2009;116(6):1097-105.

\section{Gedde 2012}

Gedde SJ, Herndon LW, Brandt JD, Budenz DL, Feuer WJ, Schiffman JC, et al. Postoperative complications in the Tube Versus Trabeculectomy (TVT) study during five years of followup. American Journal of Ophthalmology 2012;153(5):804-14.

\section{Glanville 2006}

Glanville JM, Lefebvre C, Miles JN, Camosso-Stefinovic J. How to identify randomized controlled trials in MEDLINE: ten years on. Journal of the Medical Library Association 2006;94(2):130-6.

\section{Heijl 2002}

Heijl A, Leske MC, Bengtsson B, Hyman L, Bengtsson B, Hussein $M$, et al. Reduction of intraocular pressure and glaucoma progression: results from the Early Manifest Glaucoma Trial. Archives of Ophthalmology 2002;120(10):1268-79.

\section{Higgins 2017}

Higgins JPT, Altman DG, Sterne JAC (editors). Chapter 8: Assessing risk of bias in included studies. In: Higgins JPT, Churchill R, Chandler J, Cumpston MS (editors), Cochrane Handbook for Systematic Reviews of Interventions version 5.2.0 (updated June 2017), Cochrane, 2017. Available from www.training.cochrane.org/handbook.

\section{Ismail 2015}

Ismail R, Azuara-Blanco A, Ramsay CR. Outcome measures in glaucoma: a systematic review of Cochrane Reviews and Protocols. Journal of Glaucoma 2015;24(7):533-8.

\section{Ismail 2016}

Ismail R, Azuara-Blanco A, Ramsay CR. Consensus on outcome measures for glaucoma effectiveness trials: results from a delphi and nominal group technique approaches. Journal of Glaucoma 2016;25(6):539-46.

\section{James Lind Alliance 2013}

James Lind Alliance Sight Loss and Vision Priority Setting Partnership. Setting Priorities for Eye Research. www.sightlosspsp.org.uk (accessed 10 June 2014).

\section{Kaplowitz 2016}

Kaplowitz K, Bussel II, Honkanen R, Schuman JS, Loewen NA. Review and meta-analysis of ab-interno trabeculectomy outcomes. British Journal of Ophthalmology 2016;100(5):594-600.

\section{Francis 2011}

Francis BA, Singh K, Lin SC, Hodapp E, Jampel HD, Samples JR, et al. Novel glaucoma procedures: a report by 


\section{Kass 2002}

Kass MA, Heuer DK, Higginbotham EJ, Johnson CA, Keltner JL, Miller JP, et al. The Ocular Hypertension Treatment Study: a randomized trial determines that topical ocular hypotensive medication delays or prevents the onset of primary open-angle glaucoma. Archives of Ophthalmology 2002;120(6):701-13.

\section{King 2013}

King A, Azuara-Blanco A, Tuulonen A. Glaucoma. BMJ 2013;346:f3518.

\section{King 2018}

King AJ, Shah A, Nikita E, Hu K, Mulvaney CA, Stead R, et al. Subconjunctival draining minimally-invasive glaucoma devices for medically uncontrolled glaucoma. Cochrane Database of Systematic Reviews 2018, Issue 12. Art. No: CD012742. [DOI: 10.1002/14651858.CD012742.pub2]

\section{Kirwan 2013}

Kirwan JF, Lockwood AJ, Shah P, Macleod A, Broadway DC, King AJ, et al. Trabeculectomy in the 21st century: a multicenter analysis. Ophthalmology 2013;120(12):2532-9.

\section{Langendam 2013}

Langendam MW, Akl EA, Dahm P, Glasziou P, Guyatt G, Schünemann HJ. Assessing and presenting summaries of evidence in Cochrane reviews. Systematic Reviews 2013;2:81.

\section{Le 2019}

Le JT, Bicket AK, Wang L, Li T. Ab interno trabecular bypass surgery with iStent for open-angle glaucoma. Cochrane Database of Systematic Reviews 2019, Issue 3. Art. No: CD012743. [DOI: 10.1002/14651858.CD012743.pub2]

\section{Lichter 2001}

Lichter PR, Musch DC, Gillespie BW, Guire KE, Janz NK, Wren PA, et al. Interim clinical outcomes in the Collaborative Initial Glaucoma Treatment Study comparing initial treatment randomized to medications or surgery. Ophthalmology 2001;108(11):1943-53.

\section{Mansberger 2012}

Mansberger SL, Gordon MO, Jampel H, Bhorade A, Brandt JD, Wilson $\mathrm{B}$, et al. Reduction in intraocular pressure after cataract extraction: the Ocular Hypertension Treatment Study. Ophthalmology 2012;119(9):1826-31.

\section{Minckler 2005}

Minckler DS, Baerveldt G, Alfaro MR, Francis BA. Clinical results with the Trabectome for treatment of open-angle glaucoma. Ophthalmology 2005;112(6):962-7.

\section{Mosaed 2014}

Mosaed S. The first decade of global Trabectome outcomes. Clinical and Surgical Ophthalmology 2014;32(1):21-9.

\section{Okeke 2009}

Okeke CO, Quigley HA, Jampel HD, Ying GS, Plyler RJ, Jiang Y, et al. Adherence with topical glaucoma medication monitored electronically: the Travatan Dosing Aid study. Ophthalmology 2009;116(2):191-9.

\section{Overby 2009}

Overby DR, Stamer WD, Johnson M. The changing paradigm of outflow resistance generation: towards synergistic models of the JCT and inner wall endothelium. Experimental Eye Research 2009;88(4):656-70.

\section{Peters 2013}

Peters D, Bengtsson B, Heijl A. Lifetime risk of blindness in open-angle glaucoma. American Journal of Ophthalmology 2013;156(4):724-30.

\section{Piltz 2000}

Piltz J, Gross R, Shin DH, Beiser JA, Dorr DA, Kass MA, et al. Contralateral effect of topical beta-adrenergic antagonists in initial one-eyed trials in the ocular hypertension treatment study. American Journal of Ophthalmology 2000;130(4):441-53.

\section{Quigley 2006}

Quigley HA, Broman AT. The number of people with glaucoma worldwide in 2010 and 2020. British Journal of Ophthalmology 2006;90(3):262-7.

\section{Radcliffe 2010}

Radcliffe NM, Musch DC, Niziol LM, Liebmann JM, Ritch R, Collaborative Initial Glaucoma Treatment Study Group. The effect of trabeculectomy on intraocular pressure of the untreated fellow eye in the collaborative initial glaucoma treatment study. Ophthalmology 2010;117(11):2055-60.

\section{Review Manager 2014 [Computer program]}

Nordic Cochrane Centre, The Cochrane Collaboration Review Manager 5 (RevMan 5). Version 5.3. Copenhagen: Nordic Cochrane Centre, The Cochrane Collaboration, 2014.

\section{Sandhu 2017}

Sandhu A, Jayaram H, Hu K, Bunce C, Gazzard G. Ab interno supraciliary microstent surgery for open angle glaucoma. Cochrane Database of Systematic Reviews 2017, Issue 9. Art. No: CD012802. [DOI: 10.1002/14651858.CD012802]

\section{Tóth 2017}

Tóth M, Shah A, Hu K, Bunce C, Gazzard G. Endoscopic cyclophotocoagulation (ECP) for open angle glaucoma and primary angle closure. Cochrane Database of Systematic Reviews 2019, Issue 2. Art. No: CD012741. [DOI: 10.1002/14651858.CD012741.pub2]

\section{References to other published versions of this review}

\section{Hu 2015}

Hu K, Gazzard G, Bunce C, Wormald R. Ab interno trabecular bypass surgery with Trabectome for open angle glaucoma. Cochrane Database of Systematic Reviews 2015, Issue 5. Art. No: CD011693. [DOI: 10.1002/14651858.CD011693]

\section{Hu 2016}

Hu K, Gazzard G, Bunce C, Wormald R. Ab interno trabecular bypass surgery with Trabectome for open angle glaucoma. Cochrane Database of Systematic Reviews 2016, Issue 8. Art. No: CD011693. [DOI: 10.1002/14651858.CD011693.pub2] 


\section{CHARACTERISTICS OF STUDIES}

Characteristics of included studies [ordered by study ID]

Ting 2018

\section{Study characteristics}

Methods Single-centre, single-surgeon, parallel-group randomised controlled trial with 2 study arms

1 eye included in study (method of selection not specified)

Country: Canada
Total number of participants randomised: $\mathrm{n}=19$
Number of men and women: $6 / 10$ men, $4 / 10$ women (phaco-AIT); $4 / 9$ men, $5 / 9$ women (phaco-Trab)
Age, years, mean \pm SD: $71.3 \pm 6.3$ (phaco-AIT); $67.4 \pm 5.9$ (phaco-Trab)
Inclusion criteria (which were 'expanded during the study'...'to improve enrollment'):
1. age 40 to 85 years
2. open-angle glaucoma (including pseudoexfoliative glaucoma)
3. open-angles ( $\geq$ Shaffer grade II)
4. inadequately controlled IOP requiring surgical intervention and/or glaucoma/IOP on tolerated med-
ical therapy (latter from trial report)
5. visually significant cataract
6. willing to complete quality of life questionnaires
7. capable of informed consent and available for at least 1 year follow-up

\section{Exclusion criteria:}

1. any form of angle-closure glaucoma

2. secondary open-angle glaucomas (except pseudoexfoliative glaucoma)

3. absence of clear angle landmarks on gonioscopy

4. other ocular disease that may affect assessments of visual acuity, visual fields, or tonometry

5. previous angle or filtering surgery

6. steroid use within the past 3 months

7. presence of significant comorbidities (in original protocol but not mentioned in the trial report)

Interventions

Intervention: phaco-AIT. Combined Trabectome and cataract extraction with intraocular lens insertion $(n=10)$

Comparator: phaco-Trab. Combined trabeculectomy with mitomycin $\mathrm{C}$ and cataract extraction with intraocular lens insertion $(n=9)$

Outcomes

\section{Primary outcome measures:}

- mean IOP at 6 months (per protocol)

- surgical complication rates (time frame: intraoperative and postoperative up to 12 months) (per protocol). These were divided into early ( $<30$ days postoperative) and late ( $>30$ days postoperative) and further categorised into mild, moderate, and severe

\section{Secondary outcome measures:}

- mean difference in IOP from baseline to 6 months (per protocol - reported in Table 3)

- target IOP (not in original protocol)

- mean IOP at 12 months (per protocol) 
Ting 2018 (Continued)

- quality of life measures (time frame: preoperative and postoperative at 6 and 12 months) (planned outcome, but not reported)

- mean number of glaucoma medications (time frame: 12 months) (per protocol)

- visual acuity (time frame: 12 months) (planned outcome, but not reported)

- need for additional laser (excluding suture lysis) and surgical interventions (time frame: 12 months) (per protocol)

IOP at baseline (Mean \pm SD, $\mathbf{m m H g}$ ): $20.0 \pm 5.3$ (phaco-AIT), $23.1 \pm 6.4$ (phaco-Trab)

Follow-up: 1 day, 1 week, and then 1, 3, 6, and 12 months

Adverse events reported $(\mathrm{Y} / \mathrm{N}): \mathrm{Y}$

\section{Notes}

\section{Trial registration: NCT00901108}

Date conducted: Participants were recruited from July 2009 to October 2014. Originally intended to recruit 52 participants ( 26 participants per study arm). However, recruitment was closed early (total recruitment $=19$ ) owing to 'clearer indications for each technique over time leading to lack of clinical equipoise essential for patient randomization/recruitment'.

Declaration of interests: 'The authors have no proprietary or commercial interest in any materials discussed in this article'.

Funding sources: University of Alberta, Faculty of Medicine and Dentistry for initial study start-up grant.

\section{Risk of bias}

\begin{tabular}{|c|c|c|}
\hline Bias & Authors' judgement & Support for judgement \\
\hline $\begin{array}{l}\text { Random sequence genera- } \\
\text { tion (selection bias) }\end{array}$ & Low risk & $\begin{array}{l}\text { 'Participants were block randomized to either phaco-AIT or phaco-Trab using a } \\
\text { randomly permutated block scheme'. }\end{array}$ \\
\hline $\begin{array}{l}\text { Allocation concealment } \\
\text { (selection bias) }\end{array}$ & Low risk & $\begin{array}{l}\text { 'Study enrollment and randomization were performed by a study coordinator } \\
\text { to ensure allocation concealment'. }\end{array}$ \\
\hline $\begin{array}{l}\text { Blinding of participants } \\
\text { and personnel (perfor- } \\
\text { mance bias) } \\
\text { All outcomes }\end{array}$ & Unclear risk & The trial report does not state if participants and personnel were masked. \\
\hline $\begin{array}{l}\text { Blinding of outcome as- } \\
\text { sessment (detection bias) } \\
\text { All outcomes }\end{array}$ & Unclear risk & $\begin{array}{l}\text { The trial report does not state if outcome assessors were masked to the treat- } \\
\text { ment assignments. }\end{array}$ \\
\hline $\begin{array}{l}\text { Incomplete outcome data } \\
\text { (attrition bias) } \\
\text { All outcomes }\end{array}$ & High risk & $\begin{array}{l}\text { One of } 9 \text { participants in the phaco-Trab group was lost to follow-up by } 6 \\
\text { months. One of } 10 \text { participants in the phaco-AIT group missed follow-up at } 6 \\
\text { months but returned to follow-up at } 12 \text { months. }\end{array}$ \\
\hline $\begin{array}{l}\text { Selective reporting (re- } \\
\text { porting bias) }\end{array}$ & High risk & $\begin{array}{l}\text { Quality of life measure and visual acuity were planned to be reported accord- } \\
\text { ing to the original protocol, but were not included in the trial report. Achieve- } \\
\text { ment of target IOP was reported, but this outcome measure was not planned } \\
\text { in the protocol. }\end{array}$ \\
\hline Other bias & High risk & $\begin{array}{l}\text { The original protocol described a planned sample size of } 52 \text { ( } 26 \text { per study arm). } \\
\text { However, the trial report describes a planned sample size of } 26 \text { (13 per group). } \\
\text { The study was terminated before the intended sample size was reached 'due } \\
\text { to slow recruitment and increasing lack of clinical equipoise over time'. }\end{array}$ \\
\hline
\end{tabular}


IOP: intraocular pressure

Characteristics of excluded studies [ordered by study ID]

\begin{tabular}{ll}
\hline Study & Reason for exclusion \\
\hline NCT03894631 & $\begin{array}{l}\text { Randomised controlled study but within-person study: intervention and comparator are in the } \\
\text { same participant. }\end{array}$ \\
\hline
\end{tabular}

Characteristics of ongoing studies [ordered by study ID]

JPRN-UMIN000030167

\begin{tabular}{ll}
\hline Study name & Trabectome versus microhook ab interno Trabeculotomy comparative study (TramTrac Study) \\
\hline Methods & Multicentre, open, randomised controlled noninferiority trial \\
\hline Participants & Ge: 20 to 90 years \\
Target sample size: $\mathrm{n}=120$ \\
Inclusion criteria: \\
- open anterior chamber angle with Shaffer classification grade III or IV \\
- ocular hypertension, primary open-angle glaucoma, exfoliation syndrome, or exfoliation glauco- \\
ma \\
- at screening, IOP is reproducibly equal to or more than 20 and less than 40 mmHg under at least \\
one antiglaucoma medication use \\
- no history of intraocular surgery other than cataract surgery \\
mean deviation better than - $12 \mathrm{~dB}$, and two or less juxtafoveal points with $0 \mathrm{~dB}$ on the Humphrey $30-2$ or $24-2$ program or equivalent \\
- a person who voluntarily provides us a written informed consent to participate in this study
\end{tabular}

\section{Exclusion criteria:}

- a person with only one functional eye

- inapplicability to Goldmann applanation tonometry

- presence or a history of uveitis

- a person who received oral, topical, or subTenon injection of corticosteroid within 6 months

- presence or a history of diabetes

- a pacemaker recipient

- a person who received cataract surgery on the nominated eye within 6 months

- a history of laser trabeculoplasty or laser iridotomy

- corneal endothelial density $<1000 / \mathrm{mm}^{2}$

- a pregnant or breast-feeding woman or a woman who plans to be pregnant

- an individual who participated in other studies within 4 months

- a person judged to be ineligible for any reason by a doctor in charge
Intervention: Trabectome $(\mathrm{n}=60)$

Comparator: Ab interno trabeculectomy using microhook $(n=60)$ 
Definition: Surgery is defined as success if any 'event' described below does not happen at one month after surgery. Then, the duration of treatment success is defined as a duration from the operation day to a time when any one of the four events happens in the eye with surgery success.

Event:

- at two consecutive visits, IOP exceeds $21 \mathrm{mmHg}$ or does not reach $5 \mathrm{mmHg}$

- IOP reduction rate compared with baseline does not reach $20 \%$

- glaucoma medical therapy is augmented

- additional glaucoma surgery is required

\section{Secondary outcomes:}

Efficacy outcomes:

- duration of treatment success defined by more stringent criteria. Thus, event 1 is defined as that of two consecutive visits, IOP exceeds $18 \mathrm{mmHg}$ or does not reach $5 \mathrm{mmHg}$. Other three events are the same as those used in the primary outcome measure

- pre- and postoperative IOP at 1, 3,6, and 12 months

- the number of pre- and postoperative antiglaucoma medications

- success or failure of surgery at 12 months

- presence or absence of additional glaucoma surgery

Safety outcomes:

- presence, amount, and duration of hyphema

- presence, amount, and duration of transient postoperative IOP spike

- corneal endothelial density at 12 months after surgery

- other adverse events

Other outcomes (exploratory outcomes):

- presence and degree of peripheral anterior synechiae

- final IOP

Notes Sponsor: Department of Ophthalmology, Kobe University Hospital, Japan

IOP: intraocular pressure

\section{DATA AND ANALYSES}




\section{Comparison 1. phaco-AlT versus phaco-Trab}

\begin{tabular}{|c|c|c|c|c|}
\hline Outcome or subgroup title & $\begin{array}{l}\text { No. of } \\
\text { studies }\end{array}$ & $\begin{array}{l}\text { No. of } \\
\text { partici- } \\
\text { pants }\end{array}$ & Statistical method & Effect size \\
\hline $\begin{array}{l}\text { 1.1 Proportion of participants who were medication-free at } \\
12 \text { months }\end{array}$ & 1 & & $\begin{array}{l}\text { Odds Ratio (M-H, Fixed, } \\
95 \% \mathrm{Cl})\end{array}$ & $\begin{array}{l}\text { Subtotals } \\
\text { only }\end{array}$ \\
\hline 1.2 Mean change in IOP at 12 months & 1 & & $\begin{array}{l}\text { Mean Difference (IV, Fixed, } \\
95 \% \mathrm{CI} \text { ) }\end{array}$ & $\begin{array}{l}\text { Totals not } \\
\text { selected }\end{array}$ \\
\hline $\begin{array}{l}\text { 1.3 Mean change in number of IOP-lowering medications per } \\
\text { day }\end{array}$ & 1 & & $\begin{array}{l}\text { Mean Difference (IV, Fixed, } \\
95 \% \mathrm{CI})\end{array}$ & $\begin{array}{l}\text { Totals not } \\
\text { selected }\end{array}$ \\
\hline $\begin{array}{l}\text { 1.4 Proportion of participants who achieved an IOP of } 14 \\
\mathrm{mmHg} \text { or less at } 12 \text { months }\end{array}$ & 1 & & $\begin{array}{l}\text { Odds Ratio (M-H, Fixed, } \\
95 \% \mathrm{Cl})\end{array}$ & $\begin{array}{l}\text { Subtotals } \\
\text { only }\end{array}$ \\
\hline $\begin{array}{l}\text { 1.5 Proportion of participants who achieved an IOP of } 17 \\
\mathrm{mmHg} \text { or less at } 12 \text { months }\end{array}$ & 1 & & $\begin{array}{l}\text { Odds Ratio (M-H, Fixed, } \\
95 \% \mathrm{Cl})\end{array}$ & $\begin{array}{l}\text { Subtotals } \\
\text { only }\end{array}$ \\
\hline $\begin{array}{l}\text { 1.6 Proportion of participants who achieved an IOP of } 21 \\
\mathrm{mmHg} \text { or less at } 12 \text { months }\end{array}$ & 1 & & $\begin{array}{l}\text { Odds Ratio (M-H, Fixed, } \\
95 \% \mathrm{Cl})\end{array}$ & $\begin{array}{l}\text { Subtotals } \\
\text { only }\end{array}$ \\
\hline $\begin{array}{l}\text { 1.7 Proportion of participants who required further glauco- } \\
\text { ma surgery, including laser, at } 12 \text { months }\end{array}$ & 1 & & $\begin{array}{l}\text { Odds Ratio (M-H, Fixed, } \\
95 \% \mathrm{Cl})\end{array}$ & $\begin{array}{l}\text { Subtotals } \\
\text { only }\end{array}$ \\
\hline $\begin{array}{l}\text { 1.8 Proportion of participants experiencing any intra- and } \\
\text { postoperative complications }\end{array}$ & 1 & & $\begin{array}{l}\text { Odds Ratio (M-H, Fixed, } \\
95 \% \mathrm{Cl})\end{array}$ & $\begin{array}{l}\text { Subtotals } \\
\text { only }\end{array}$ \\
\hline $\begin{array}{l}\text { 1.9 Proportion of participants experiencing intra- and post- } \\
\text { operative complications within } 30 \text { days postoperative }\end{array}$ & 1 & & $\begin{array}{l}\text { Odds Ratio (M-H, Fixed, } \\
95 \% \mathrm{Cl})\end{array}$ & $\begin{array}{l}\text { Subtotals } \\
\text { only }\end{array}$ \\
\hline $\begin{array}{l}\text { 1.10 Proportion of participants experiencing intra- and post- } \\
\text { operative complications }>30 \text { days postoperative }\end{array}$ & 1 & & $\begin{array}{l}\text { Odds Ratio (M-H, Fixed, } \\
95 \% \mathrm{Cl})\end{array}$ & $\begin{array}{l}\text { Subtotals } \\
\text { only }\end{array}$ \\
\hline
\end{tabular}

Analysis 1.1. Comparison 1: phaco-AIT versus phaco-Trab, Outcome 1: Proportion of participants who were medication-free at 12 months

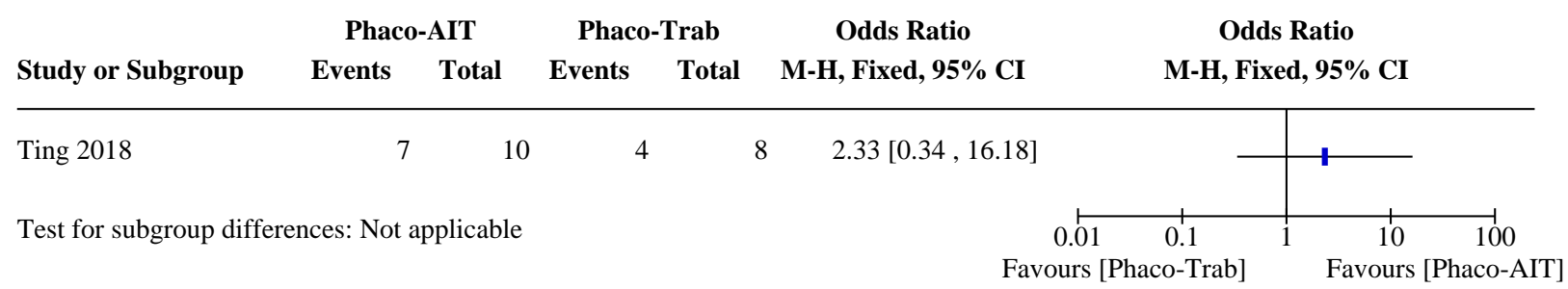


Analysis 1.2. Comparison 1: phaco-AIT versus phaco-Trab, Outcome 2: Mean change in IOP at 12 months

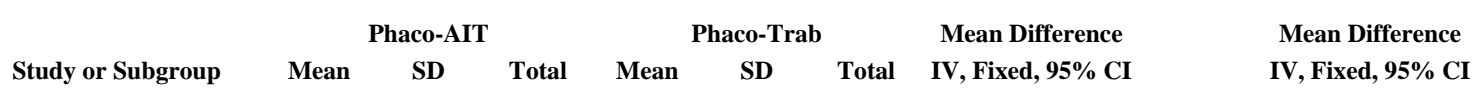

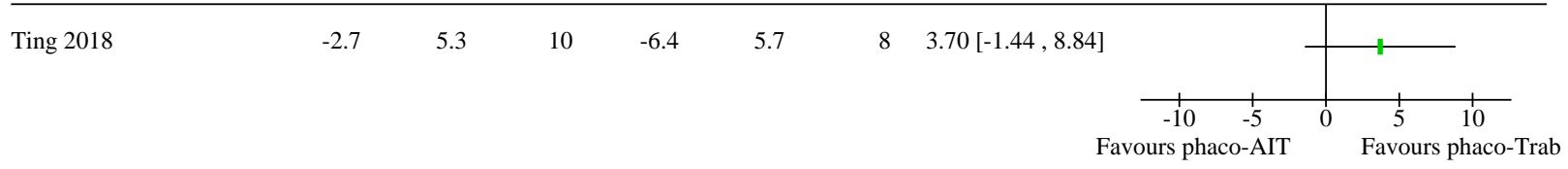

Analysis 1.3. Comparison 1: phaco-AlT versus phaco-Trab, Outcome 3: Mean change in number of IOP-lowering medications per day

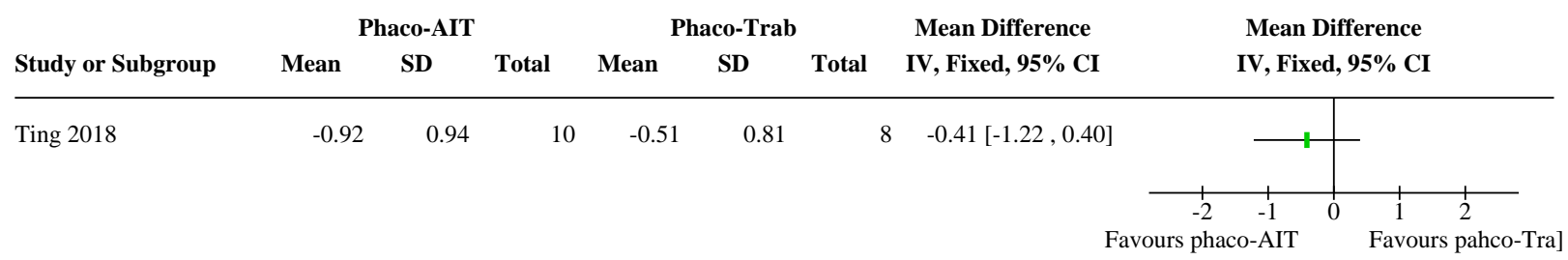

Analysis 1.4. Comparison 1: phaco-AIT versus phaco-Trab, Outcome 4: Proportion of participants who achieved an IOP of $14 \mathrm{mmHg}$ or less at 12 months

\begin{tabular}{ccccccc} 
& \multicolumn{2}{c}{ Phaco-AIT } & \multicolumn{2}{c}{ Phaco-Trab } & Odds Ratio & Odds Ratio \\
Study or Subgroup & Events & Total & Events & Total & M-H, Fixed, 95\% CI & M-H, Fixed, 95\% CI
\end{tabular}

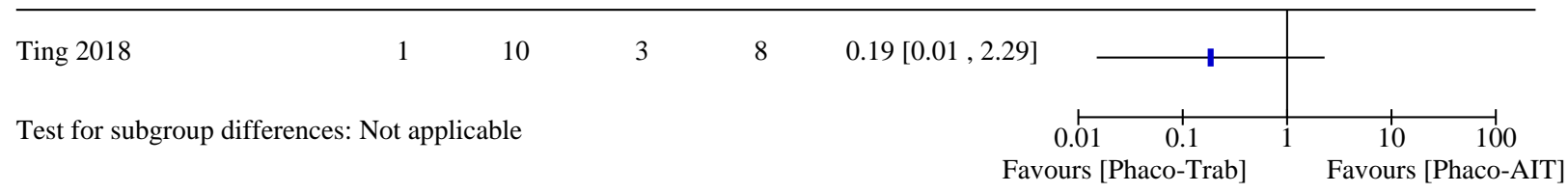

Analysis 1.5. Comparison 1: phaco-AIT versus phaco-Trab, Outcome 5: Proportion of participants who achieved an IOP of $17 \mathrm{mmHg}$ or less at 12 months

Phaco-AIT Phaco-Trab Odds Ratio Odds Ratio

Study or Subgroup Events Total Events Total M-H, Fixed, 95\% CI $\quad$ M-H, Fixed, 95\% CI

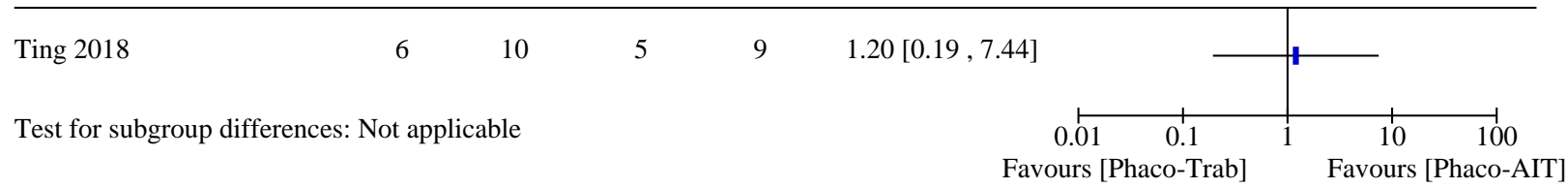


Analysis 1.6. Comparison 1: phaco-AIT versus phaco-Trab, Outcome 6:

Proportion of participants who achieved an IOP of $21 \mathrm{mmHg}$ or less at 12 months

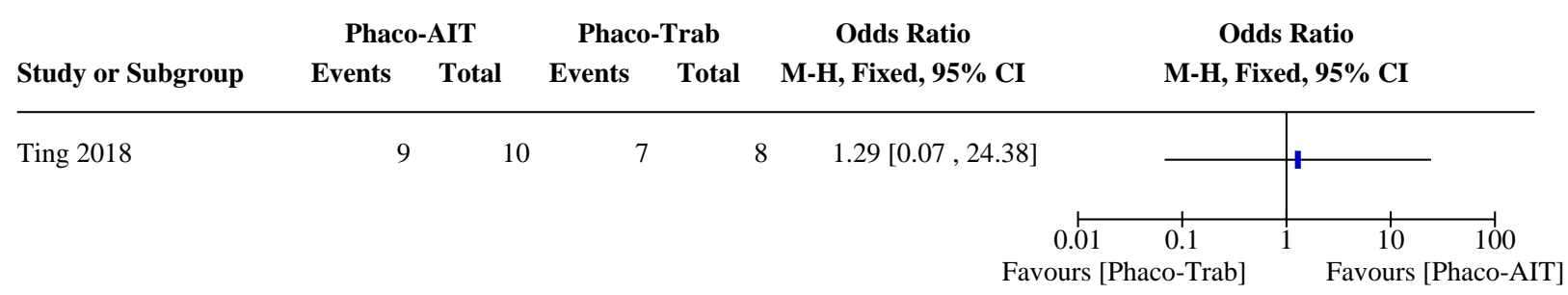

Analysis 1.7. Comparison 1: phaco-AlT versus phaco-Trab, Outcome 7: Proportion of participants who required further glaucoma surgery, including laser, at 12 months

\begin{tabular}{|c|c|c|c|c|c|c|}
\hline & Pha & AIT & Phac & Trab & Odds Ratio & Odds Ratio \\
\hline Study or Subgroup & Events & Total & Events & Total & M-H, Fixed, 95\% CI & M-H, Fixed, 95\% CI \\
\hline
\end{tabular}

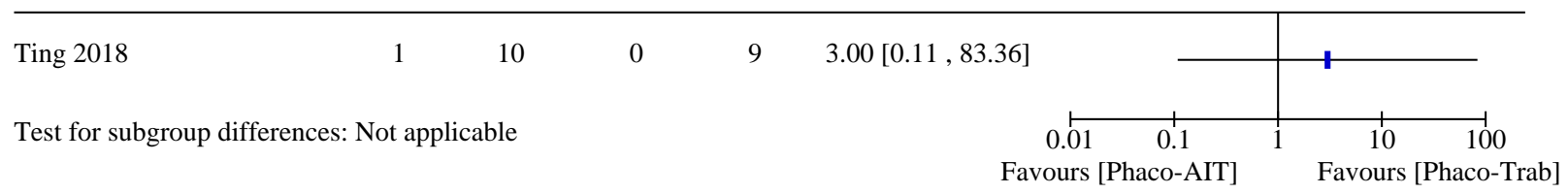

Analysis 1.8. Comparison 1: phaco-AIT versus phaco-Trab, Outcome 8: Proportion of participants experiencing any intra- and postoperative complications

\begin{tabular}{ccccccc} 
& \multicolumn{2}{c}{ Phaco-AIT } & \multicolumn{2}{c}{ Phaco-Trab } & Odds Ratio & Odds Ratio \\
Study or Subgroup & Events & Total & Events & Total & M-H, Fixed, 95\% CI & M-H, Fixed, 95\% CI
\end{tabular}

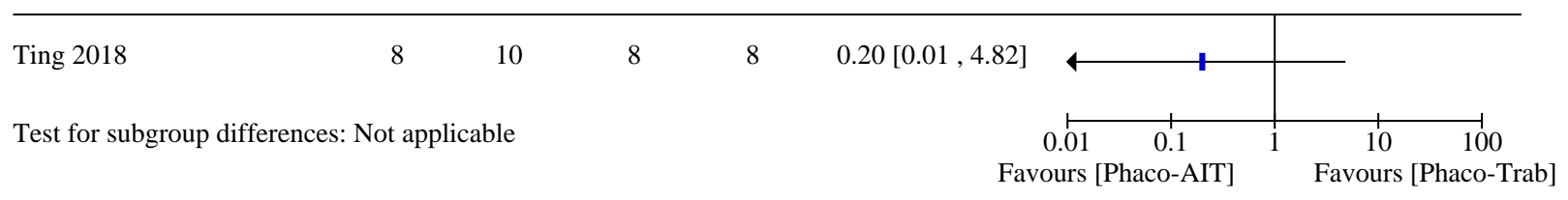

Analysis 1.9. Comparison 1: phaco-AIT versus phaco-Trab, Outcome 9: Proportion of participants experiencing intra- and postoperative complications within $\mathbf{3 0}$ days postoperative

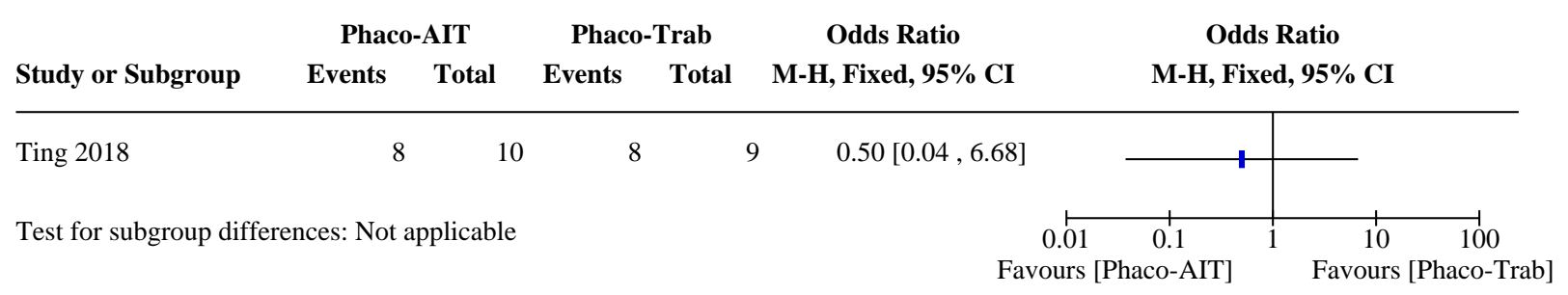


Analysis 1.10. Comparison 1: phaco-AlT versus phaco-Trab, Outcome 10: Proportion of participants experiencing intra- and postoperative complications $>30$ days postoperative

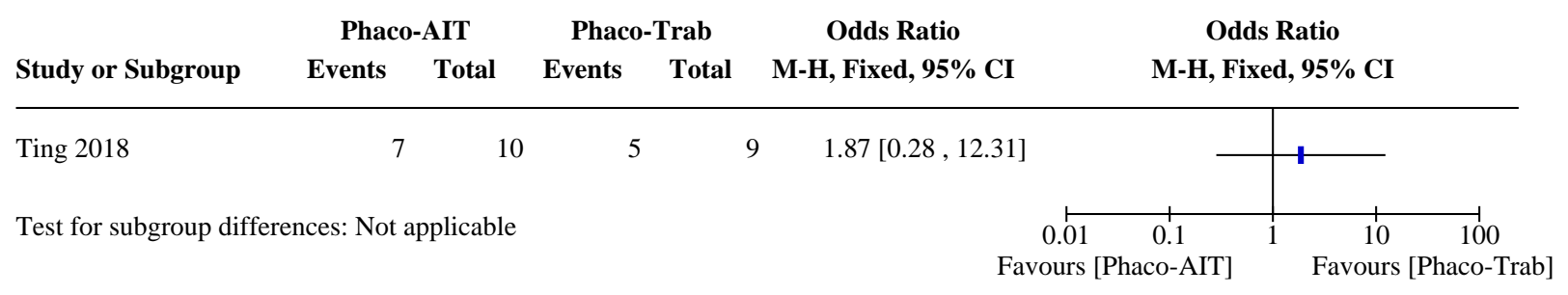

\section{APPENDICES}

\section{Appendix 1. CENTRAL search strategy}

\#1 MeSH descriptor: [Glaucoma, Open-Angle] explode all trees

\#2 MeSH descriptor: [Intraocular Pressure] explode all trees

\#3 MeSH descriptor: [Ocular Hypertension] explode all trees

\#4 OAG or POAG or IOP or OHT

\#5 simple near/3 glaucoma*

\#6 open near/2 angle near/2 glaucoma*

\#7 chronic near/2 glaucoma*

\#8 secondary near/2 glaucoma*

\#9 low near/2 tension near/2 glaucoma*

\#10 low near/2 pressure near/2 glaucoma*

\#11 normal near/2 tension near/2 glaucoma*

\#12 normal near/2 pressure near/2 glaucoma*

\#13 pigment near/2 glaucoma*

\#14 MeSH descriptor: [Exfoliation Syndrome] this term only

\#15 exfoliat ${ }^{\star}$ near/2 syndrome*

\#16 exfoliat* ${ }^{\star}$ near/2 glaucoma*

\#17 pseudoexfoliat* near/2 syndrome

\#18p seudoexfoliat* near/2 glaucoma*

$\# 19 \# 1$ or $\# 2$ or \#3 or \#4 or \#5 or \#6 or \#7 or \#8 or \#9 or \#10 or \#11 or \#12 or \#13 or \#14 or \#15 or \#16 or \#17 or \#18

\#20 Trabectome

\#21 ab interno trabeculectomy or trabeculectomy ab interno

\#22 ab interno trabeculotomy or trabeculotomy ab interno

\#23 trabecular near/2 bypass*

\#24 \#20 or \#21 or \#22 or \#23

$\# 25 \# 19$ and \#24

\section{Appendix 2. MEDLINE Ovid search strategy}

1. randomized controlled trial.pt.

2. (randomized or randomised).ab,ti.

3. placebo.ab,ti.

4. dt.fs.

5. randomly.ab,ti.

6. trial.ab,ti.

7. groups.ab,ti.

8. or/1-7

9. exp animals/

10. exp humans/

11.9 not $(9$ and 10$)$

12. 8 not 11

13. exp glaucoma open angle/

14. exp intraocular pressure/

15. ocular hypertension/

16. (OAG or POAG or IOP or OHT).tw. 
17. (simple\$ adj3 glaucoma\$).tw.

18. (open adj2 angle adj2 glaucoma\$).tw.

19. (primary adj2 glaucoma\$).tw.

20. (chronic adj2 glaucoma\$).tw.

21. (secondary adj2 glaucoma\$).tw.

22. (low adj2 tension adj2 glaucoma\$).tw.

23. (low adj2 pressure adj2 glaucoma\$).tw.

24. (normal adj2 tension adj2 glaucoma\$).tw.

25. (normal adj2 pressure adj2 glaucoma\$).tw.

26. (pigment\$ adj2 glaucoma\$).tw.

27. exfoliation syndrome/

28. (exfoliat\$ adj2 syndrome\$).tw.

29. (exfoliat\$ adj2 glaucoma\$).tw.

30. (pseudoexfoliat\$ adj2 syndrome\$).tw.

31. (pseudoexfoliat\$ adj2 glaucoma\$).tw.

32. or/13-31

33. Trabectome.tw.

34. (Ab interno adj2 (trabeculectomy or trabeculotomy)).tw.

35. (trabecular adj2 bypass\$).tw.

36. or/33-35

37. 32 and 36

38. 12 and 37

The search filter for trials at the beginning of the MEDLINE strategy is from the published paper by Glanville 2006.

\section{Appendix 3. Embase Ovid search strategy}

1. exp randomized controlled trial/

2. exp randomization/

3. exp double blind procedure/

4. exp single blind procedure/

5. random\$.tw.

6. or/1-5

7. (animal or animal experiment).sh.

8. human.sh.

9. 7 and 8

10. 7 not 9

11.6 not 10

12. exp clinical trial/

13. (clin\$ adj3 trial\$).tw.

14. ((singl\$ or doubl\$ or trebl\$ or tripl\$) adj3 (blind\$ or mask\$)).tw.

15. exp placebo/

16. placebo\$.tw.

17. random\$.tw.

18. exp experimental design/

19. exp crossover procedure/

20. exp control group/

21. exp latin square design/

22. or/ $12-21$

23. 22 not 10

24. 23 not 11

25. exp comparative study/

26. exp evaluation/

27. exp prospective study/

28. (control\$ or prospectiv\$ or volunteer\$).tw.

29. or $/ 25-28$

30. 29 not 10

31.30 not ( 11 or 23 )

32. 11 or 24 or 31

33. open angle glaucoma/

34. intraocular pressure/

35. intraocular hypertension/ 
36. (OAG or POAG or IOP or OHT).tw.

37. (open adj2 angle adj2 glaucoma\$).tw.

38. (primary adj2 glaucoma\$).tw.

39. (chronic adj2 glaucoma\$).tw.

40. (secondary adj2 glaucoma\$).tw.

41. (low adj2 tension adj2 glaucoma\$).tw.

42. (low adj2 pressure adj2 glaucoma\$).tw.

43. (normal adj2 tension adj2 glaucoma\$).tw.

44. (normal adj2 pressure adj2 glaucoma\$).tw.

45. (pigment\$ adj2 glaucoma\$).tw.

46. exfoliation syndrome/

47. (exfoliat\$ adj2 syndrome\$).tw.

48. (exfoliat\$ adj2 glaucoma\$).tw.

49. (pseudoexfoliat\$ adj2 syndrome\$).tw.

50. (pseudoexfoliat\$ adj2 glaucoma\$).tw.

51. or/33-50

52. Trabectome.tw.

53. (ab interno adj2 (trabeculectomy or trabeculotomy)).tw.

54. (trabecular adj2 bypass\$).tw.

55. or/52-54

56.51 and 55

57.32 and 56

\section{Appendix 4. ISRCTN search strategy}

Trabectome OR trabecular bypass OR ab interno trabeculectomy OR ab interno trabeculotomy

\section{Appendix 5. ClinicalTrials.gov search strategy}

(Trabectome OR trabecular bypass OR ab interno trabeculectomy OR ab interno trabeculotomy)

\section{Appendix 6. WHO ICTRP search strategy}

Trabectome OR trabecular bypass OR ab interno trabeculectomy OR ab interno trabeculotomy = Intervention

\section{Appendix 7. Data on study characteristics}

\begin{tabular}{|c|c|c|}
\hline \multicolumn{2}{|l|}{ Mandatory items } & \multirow[t]{2}{*}{ Optional items } \\
\hline Methods & & \\
\hline \multirow[t]{5}{*}{ Study design } & - Parallel-group RCT i.e. people randomised to treatment & Number of study arms \\
\hline & - Within-person RCT i.e. eyes randomised to treatment & Method of randomisation \\
\hline & - Cluster RCT i.e. communities randomised to treatment & Exclusions after randomisation \\
\hline & - Crossover RCT & Losses to follow-up \\
\hline & - Other, specify & Number randomised/analysed \\
\hline Eyes & - 1 eye included in study, specify how eye selected & Method of masking \\
\hline \multirow[t]{4}{*}{$\begin{array}{l}\text { Unit of randomisa- } \\
\text { tion/unit of analysis }\end{array}$} & $\begin{array}{l}\text { - } 2 \text { eyes included in study, both eyes received same treatment, } \\
\text { briefly specify how analysed (best/worst/average/both and adjusted } \\
\text { for within-person correlation/both and not adjusted for within-person }\end{array}$ & $\begin{array}{l}\text { How were missing data handled? } \\
\text { e.g. available-case analysis, imputa- } \\
\text { tion methods }\end{array}$ \\
\hline & correlation) and specify if mixture of 1 eye and 2 eyes & Reported power calculation $(\mathrm{Y} / \mathrm{N})$, if \\
\hline & - 2 eyes included in study, eyes received different treatments, & yes, sample size and power \\
\hline & specify if correct pair-matched analysis done & Unusual study design/issues \\
\hline
\end{tabular}


(Continued)

\section{Participants}

\begin{tabular}{|c|c|c|}
\hline Country & - & Setting \\
\hline Total number of par- & \multirow{5}{*}{$\begin{array}{l}\text { This information should be collected for total study population recruit- } \\
\text { ed into the study. If these data are reported for the people who were } \\
\text { followed up only, please indicate }\end{array}$} & Ethnic group \\
\hline ticipants & & Method of recruitment \\
\hline $\begin{array}{l}\text { Number }(\%) \text { of men } \\
\text { and women }\end{array}$ & & Participation rate \\
\hline Average age and age & & $\begin{array}{l}\text { Equivalence of baseline characteris- } \\
\text { tics }(\mathrm{Y} / \mathrm{N})\end{array}$ \\
\hline range & & Diagnostic criteria \\
\hline Inclusion criteria & - & \\
\hline Exclusion criteria & - & \\
\hline
\end{tabular}

\section{Interventions}

\begin{tabular}{lll}
\hline Intervention $(\mathrm{n}=)$ & $\cdot$ Number of people randomised to this group & $\begin{array}{l}\text { Trabectome surgical parameters, } \\
\text { e.g. degrees of meshwork ablated, } \\
\text { electrosurgical power }\end{array}$ \\
& $\cdot$ Intervention name & $\begin{array}{l}\text { Comparator parameters, e.g. } \\
\text { dosage of drugs }\end{array}$ \\
& $\cdot$ Comparator name $(\mathrm{n}=)$ & \\
& formed at same time as intervention & \\
\hline
\end{tabular}

\section{Outcomes}

\begin{tabular}{ll}
\hline $\begin{array}{l}\text { Primary and sec- } \\
\text { ondary outcomes as } \\
\text { defined in study re- } \\
\text { ports }\end{array}$ & $\cdot$ IOP at baseline \\
& $\cdot$ IOP at follow-up \\
$\cdot$ & Number of glaucoma medications at baseline \\
$\cdot$ & Number of glaucoma medications at follow-up \\
$\cdot$ & Intraoperative complications \\
$\cdot$ Postoperative complications or secondary surgery & Plannellow-up \\
$\cdot$ Duration of follow-up & $\cdot$ Loss to follow-up \\
$\cdot$ Intervals at which outcomes assessed & Adverse events reported $(\mathrm{Y} / \mathrm{N})$
\end{tabular}

\section{Notes}

\begin{tabular}{lll}
\hline Date conducted & Specify dates of recruitment of participants $\mathrm{mm} / \mathrm{yr}$ to $\mathrm{mm} / \mathrm{yr}$ & Full study name: (if applicable) \\
\hline Sources of funding & - & Date of publication \\
\hline $\begin{array}{l}\text { Declaration of inter- } \\
\text { est }\end{array}$ & Reported subgroup analyses ( $\mathrm{Y} / \mathrm{N})$ \\
\hline
\end{tabular}


WHAT'S NEW

\begin{tabular}{lll}
\hline Date & Event & Description \\
\hline 5 January 2021 & $\begin{array}{l}\text { New citation required and conclusions } \\
\text { have changed }\end{array}$ & $\begin{array}{l}\text { Results from the ongoing study have now been published and } \\
\text { included in this update (Ting 2018). One new ongoing study has } \\
\text { been included (JPRN-UMIN000030167). Two new co-authors (A } \\
\text { Shah and G Virgili) joined the team. }\end{array}$ \\
\end{tabular}

\section{HISTORY}

Protocol first published: Issue 5, 2015

Review first published: Issue 8, 2016

\section{CONTRIBUTIONS OF AUTHORS}

\section{Protocol}

Kuang Hu and Catey Bunce wrote the protocol. Kuang Hu, Catey Bunce, Gus Gazzard, and Richard Wormald reviewed and approved the protocol.

\section{Review}

Gus Gazzard and Kuang Hu screened the search results. Kuang Hu wrote the review. Kuang Hu, Catey Bunce, Gus Gazzard, and Richard Wormald reviewed and approved the review.

\section{1 update}

Anupa Shah and Kuang Hu screened the search results.

Kuang Hu wrote the review with assistance from Anupa Shah, Gianni Virgili, and Catey Bunce.

Guz Gazzard approved the final draft.

\section{DECLARATIONS OF INTEREST}

The review authors are seeking funding to address the subject of this review.

KH has performed Trabectome surgery. He has delivered a lecture entitled "Constructing clinical trials for MIGS - the lack of evidence and what to do about it" at the Moorfields International Glaucoma Symposium 2016. The Symposium was sponsored by Laboratoires Thea, which contributed an educational grant to Moorfields Eye Hospital.

AS: none known

GV: none known

CB: none known

GG has in the last five years received travel funding and his host organisation has received both educational and unrestricted research funding from pharmaceutical and equipment manufacturers that are involved in the treatment of glaucoma but none that are otherwise related to (or competing with) the subject of this review.

\section{SOURCES OF SUPPORT}

\section{Internal sources}

- National Institute for Health Research (NIHR), UK

CB acknowledges financial support for her Cochrane Eyes and Vision (CEV) research sessions from the Department of Health through the award made by the NIHR to Moorfields Eye Hospital NHS Foundation Trust and UCL Institute of Ophthalmology for a Specialist Biomedical Research Centre for Ophthalmology.

GG acknowledges support for this research by the National Institute for Health Research (NIHR) Biomedical Research Centre based at Moorfields Eye Hospital NHS Foundation Trust and UCL Institute of Ophthalmology.

The views expressed are those of the authors and not necessarily those of the NHS, the NIHR or the Department of Health. 


\section{External sources}

- National Institute for Health Research (NIHR), UK

* Richard Wormald, Co-ordinating Editor for Cochrane Eyes and Vision (CEV) acknowledged financial support for his CEV research sessions (during this update) from the Department of Health through the award made by the NIHR to Moorfields Eye Hospital NHS Foundation Trust and UCL Institute of Ophthalmology for a Specialist Biomedical Research Centre for Ophthalmology.

* The 2021 update was supported by the NIHR, via Cochrane Infrastructure funding to the CEV UK editorial base.

The views and opinions expressed therein are those of the authors and do not necessarily reflect those of the Systematic Reviews Programme, NIHR, NHS or the Department of Health.

\section{DIFFERENCES BETWEEN PROTOCOLAND REVIEW}

2021 update

- The secondary outcomes, adverse effects, and all follow-up times have been harmonised with the other MIGS reviews that form part of this suite of reviews.

- The original published review included combination therapy with phacoemulsification as an intervention and for subgroup analysis. After discussion within the review team and MIGS Consortium, we opted to include it as a separate comparison, as this is likely to be a different indication.

- We changed the Objectives to include anyone with open-angle glaucoma instead of restricting it to just those 'whose condition is inadequately controlled with drops'.

\section{N DEX TERMS}

\section{Medical Subject Headings (MeSH)}

Glaucoma, Open-Angle [ ${ }^{\star}$ surgery]; *Phacoemulsification; Randomized Controlled Trials as Topic; Trabecular Meshwork [*surgery]; Trabeculectomy [*instrumentation] [methods]

\section{MeSH check words}

Humans 